\title{
Evolution from BCS superconductivity to Bose-Einstein condensation: Current correlation function in the broken-symmetry phase
}

\author{
N. Andrenacci ${ }^{1,2}$, P. Pieri ${ }^{1}$, and G.C. Strinati ${ }^{1}$ \\ (1) Dipartimento di Fisica, UdR INFM \\ Università di Camerino, I-62032 Camerino, Italy \\ (2) Institut de Physique, Université de Neuchatel \\ CH-2000 Neuchatel, Switzerland
}

(November 7, 2018)

\begin{abstract}
We consider the current correlation function for a three-dimensional system of fermions embedded in a homogeneous background and mutually interacting via an attractive short-range potential, below the (superconducting) critical temperature. Diagrammatic contributions in the broken-symmetry phase are identified, that yield for the (wave-vector and frequency dependent) current correlation function the fermionic BCS approximation in the weak-coupling limit and the bosonic Bogoliubov approximation in the strong-coupling limit (whereby composite bosons form as bound-fermion pairs). The temperature dependence of the superfluid density (from the BCS exponential behavior at weak coupling to a power-law behavior at strong coupling) and the form of the Pippard-like kernel at zero temperature are explicitly obtained from weak to strong coupling. Quite generally, it is shown that the Pippard-like kernel is the sum of a local (London-like) term and of a nonlocal component, the local term being dominant in the strong-coupling limit and the nonlocal component in the BCS (weak-coupling) limit. It is also shown that the range of the nonlocal component is determined by the coherence length measuring the spatial correlations of the amplitude of the order parameter, namely, the correlations among different Cooper pairs (or composite bosons), rather than between the fermionic partners of a given pair. In addition, a prescription is provided for mapping the fermionic onto the bosonic diagrammatic theories in the broken-symmetry phase, thus complementing what already done in the normal phase.
\end{abstract}

PACS numbers: 74.20.-z,74.20.Fg,74.25.Nf

\section{INTRODUCTION}

Already from London and Pippard phenomenological approaches to superconductivity, the current response to an external magnetic field played a key role in accounting for the Meissner effect. The Meissner effect was, in fact, later demonstrated explicitly within the microscopic BCS approach, by examining the behavior of the (static) current correlation function ${ }^{1,2}$.

The BCS approach as it stands applies to weakcoupling superconductors, with largely overlapping Cooper pairs. Recently, however, it has become of interest to study the evolution of a superconducting system from weak to strong coupling, with the strong-coupling limit corresponding to a system of nonoverlapping (composite) bosons ${ }^{3-9}$. The physical motivation to these works stems essentially from cuprate superconductors, whereby the small value of the (superconducting) coherence length as well as the presence of a pseudogap above the critical temperature in the underdoped region ${ }^{10,11}$ have suggested the possible relevance of a crossover scenario from a weak-coupling limit for overdoped samples (with Cooper pairs forming and condensing at the critical temperature within a BCS description) toward a strongcoupling limit for underdoped samples (with preformed (composite) bosons existing above the superconducting critical temperature and Bose-Einstein (BE) condensing below it). For cuprate superconductors, it seems actually likely that the pairing corresponds to an intermediate regime between overlapping Cooper pairs and nonoverlapping composite bosons. More generally, the possible occurrence of a crossover from BCS to BE has been emphasized for other classes of "unconventional" superconductors as well ${ }^{12}$.

The first discussion of the crossover from BCS to $\mathrm{BE}$ can be found in the pioneering paper by Eagles dealing with superconductivity in low-carrier doped superconductors ${ }^{13}$. A systematic approach to this problem was then provided by Leggett ${ }^{14}$, who showed that for a fermionic system with an attractive interaction at zero temperature a smooth crossover from a BCS ground state of overlapping Cooper pairs to a condensate of composite bosons occurs as the strength of the attraction increases. A few years later the problem was reconsidered by Nozières and Schmitt-Rink ${ }^{15}$, who extended the approach to finite temperatures by using diagrammatic methods, always finding a smooth crossover between the two limits.

Several quantities have so far been calculated within the BCS-BE crossover approach, including the critical temperature and chemical potential ${ }^{16}$, the coherence length(s) at zero temperature ${ }^{6}$, the spectral function above $^{17}$ and below ${ }^{18}$ the critical temperature, and the effect of an external magnetic field on the pseudogap tem- 
perature $^{19}$. No systematic study yet exists, however, for the current correlation function and derived quantities, such as the (temperature dependence of the) superfluid density and the Pippard kernel. Purpose of this paper is to provide a detailed calculation of these quantities, by relying on controlled approximations in the two (weakand strong-coupling) limits, which allow us to examine specifically how the response of the original Fermi system can be interpreted in terms of the response of an effective Bose system in the strong-coupling limit.

We shall rely on a diagrammatic approach that selects the relevant contributions to the (wave-vector and frequency dependent) current correlation function in the weak- and strong-coupling limits separately, and then sum these contributions to obtain the current correlation function for all couplings. The contributions selected in the strong-coupling limit will appear as fluctuation contributions when extrapolated to the weak-coupling limit. In this paper, we will consider a Fermi system with an attractive (point-contact) interparticle interaction embedded in a three-dimensional continuum, with an isotropic ( $s$-wave) gap function. The ensuing diagrammatic theory in the broken-symmetry phase will be thus simplified considerably, yet reproducing the desired features in both limits, in a similar fashion to what was done in the normal phase above the critical temperature ${ }^{9,20}$. No lattice effects nor the occurrence of an angular dependent ( $d$-wave) gap function will accordingly be taken into account, deferring their study to future work. The present approach should be regarded as a necessary preliminary step toward the development of a theory of the current response function over the whole crossover range for more realistic models.

As far as the current correlation function of interest is concerned, the two (weak- and strong-coupling) limits will be described by the BCS approximation (for fermions) and the Bogoliubov approximation (for bosons), respectively. Again, these represent the simplest approximations which can be conceived to describe fermionic and bosonic systems, respectively, in the broken-symmetry (superfluid) phase, and share analogous self-consistent constructions to generate the relevant excited states within a temperature-dependent mean-field approximation. Albeit the Bogoliubov approximation for bosons is known to suffer from several shortcomings ${ }^{21,22}$ (which could be overcome, at least in principle, by considering more involved approximations for condensed bosons ${ }^{23}$ ), we shall anyhow restrict ourselves to this approximation for the bosonic limit, since we are ultimately interested in obtaining a reasonable strong-coupling limit while following the evolution of the fermionic current correlation function from weak to strong coupling. In this respect, we should mention that the Bogoliubov approximation has been recently adopted to get the response function near zero temperature for a Bose gas in the context of the BE condensation of ultracold atomic dilute gases ${ }^{24}$.

While the BCS approximation to the current corre- lation function is well established in weak coupling ${ }^{1,2}$, recovering the Bogoliubov results for (composite) bosons from an originally fermionic theory is altogether nontrivial and will therefore be considered at some length in this paper. Specifically, we shall identify the fermionic diagrams (over and above the standard BCS "bubble" contribution) which reproduce both the longitudinal and transverse contributions to the (wave-vector and frequency dependent) Bogoliubov current correlation function, namely, the "ladder" diagrams known for making the BCS result conserving ${ }^{1}$ and the Aslamazov-Larkin (AL) type diagram(s $)^{25}$ here extended to the brokensymmetry phase, in the order. Since these diagrams can be evaluated analytically in the strong-coupling limit, a detailed account of this derivation will be provided in what follows. In this context, the prescription for mapping the fermionic onto the bosonic diagrammatic theories in the broken-symmetry phase will also be provided, in an analogous fashion to what already achieved in the normal phase ${ }^{9}$.

Besides providing a formal theory that bridges weakand strong-coupling approximations to the current correlation function, the main physical results of this paper concern, in particular, the temperature dependence of the superfluid density (from the BCS exponential behavior at weak coupling to a power-law behavior at strong coupling) and the form of the Pippard-like kernel at zero temperature $^{26}$. The contributions of the BCS and AL diagrams to the temperature dependence of the superfluid density will be numerically calculated for various couplings from weak to strong, and their relative importance assessed. [These calculations require only the static contribution to the current correlation function, so that the analytic continuation from Matsubara to real frequencies is not required.] It will be also shown that the Pippardlike kernel is the sum of a local (London-like) term and of a nonlocal component, where only the local term survives in the strong-coupling limit, thus correctly reproducing the result for non-interacting (composite) bosons, while only the nonlocal component survives in the BCS (weakcoupling) limit, as expected. It will be further shown that the characteristic length that controls the range of the nonlocal component coincides (for a clean system) with the coherence length $\xi_{\text {phase }}$ introduced in Ref. 6 as a measure of the spatial correlations of the amplitude of the order parameter, over the whole crossover range from weak to strong coupling. The length $\xi_{\text {phase }}$ relates the correlations among different Cooper pairs or between composite bosons, while correlations between fermionic partners in a pair is associated with the different lenght scale $\xi_{\text {pair. }}{ }^{6}$ The quantity $\xi_{\text {phase }}$, in turn, coincides with the Pippard coherence length $\xi_{0}$ (and with $\xi_{\text {pair }}$ ) in the weakcoupling limit, decreases as the coupling is increased from the weak-toward the intermediate-coupling regime, and eventually starts again to increase upon approaching the strong-coupling (bosonic) limit where $\xi_{\text {phase }} \gg \xi_{\text {pair }}^{6}$ (see also Ref. 27 for an extension to the lattice case).

The plan of the paper is as follows. In Section II we 
consider the current correlation function within the (conserving) BCS approximation (i.e., bubble plus ladder diagrams) and show that, in the strong-coupling limit, it maps onto the longitudinal part of the current correlation function for (composite) bosons within the Bogoliubov approximation. In Section III we identify, in addition, the contribution to the fermionic current correlation function that maps onto the transverse part of the current correlation function for (composite) bosons within the Bogoliubov approximation. In Section IV numerical results for the superfluid density vs temperature as well as for the Pippard-like kernel at zero temperature are presented, over the whole range from weak to strong coupling. Section V gives our conclusions. The Appendices discuss more technical material. Specifically, in Appendix A the connection between the particle-particle ladder in the broken-symmetry phase and the Bogoliubov propagators is obtained; in Appendix B the mapping between the fermionic and bosonic diagrammatic structures in the broken-symmetry phase is established; in Appendix $\mathrm{C}$ the form of the wave-vector and frequency dependent current correlation function for bosons within the Bogoliubov approximation is discussed, with emphasis on the ensuing form of the Pippard-like kernel; and in Appendix D the BCS bubble is examined in a novel way as to extract its kernel for all couplings and not only in the weak-coupling limit.

\section{CURRENT CORRELATION FUNCTION WITHIN THE BCS APPROXIMATION IN THE STRONG-COUPLING LIMIT}

In this Section, we reconsider the well-known BCS approximation for the current correlation function ${ }^{1}$, in the form which fulfills conservation $\operatorname{laws}^{28}$. We will specifically show that in the strong-coupling limit of the fermionic attraction (and at temperatures small compared with the dissociation energy of composite bosons) this approximation maps onto the longitudinal part of the current correlation function for bosons within the Bogoliubov approximation. This result, which is per se nontrivial, requires us to search for additional contributions to the current correlation function which, in the strong-coupling limit, reproduce the transverse part of the current correlation function for bosons within the Bogoliubov approximation, as discussed in the next Section.

Quite generally, the current correlation function at finite temperature in the imaginary time (Matsubara) representation reads ${ }^{2}$ :

$$
\chi_{\gamma, \gamma^{\prime}}\left(\mathbf{r} \tau, \mathbf{r}^{\prime} \tau^{\prime}\right)=-\left\langle T_{\tau}\left[j_{\gamma}(\mathbf{r} \tau) j_{\gamma^{\prime}}\left(\mathbf{r}^{\prime} \tau^{\prime}\right)\right]\right\rangle
$$

where $0 \leq \tau, \tau^{\prime} \leq \beta\left(\beta=1 /\left(k_{B} T\right)\right.$ being the inverse temperature and $k_{B}$ the Boltzmann's constant), $T_{\tau}$ is the imaginary-time ordering operator, $\langle\cdots\rangle$ is a thermal average, and $\gamma, \gamma^{\prime}=(x, y, z)$ label Cartesian components. For our purposes, it is convenient to write the current operator in (1) in terms of the Nambu representation of the field operators

$$
\Psi(\mathbf{r})=\left(\begin{array}{c}
\psi_{\uparrow}(\mathbf{r}) \\
\psi_{\downarrow}^{\dagger}(\mathbf{r})
\end{array}\right)
$$

as follows:

$$
\mathbf{j}(\mathbf{r})=\frac{1}{2 i m} \sum_{l=1}^{2}\left(\Psi_{\ell}^{\dagger}(\mathbf{r}) \nabla \Psi_{\ell}(\mathbf{r})-\left(\nabla \Psi_{\ell}^{\dagger}(\mathbf{r})\right) \Psi_{\ell}(\mathbf{r})\right)
$$

$m$ being the fermionic mass.

The current correlation function (1) can be expressed in terms of the single-particle Green's function

$$
\mathcal{G}(1,2)=-\left\langle T_{\tau}\left[\Psi(1) \Psi^{\dagger}(2)\right]\right\rangle
$$

and of the two-particle Green's function

$$
\mathcal{G}_{2}\left(1,2 ; 1^{\prime}, 2^{\prime}\right)=\left\langle T_{\tau}\left[\Psi(1) \Psi(2) \Psi^{\dagger}\left(2^{\prime}\right) \Psi^{\dagger}\left(1^{\prime}\right)\right]\right\rangle
$$

(with the short-hand notation $1=\left(\mathbf{r}_{1}, \tau_{1}, \ell_{1}\right)$ and so on, in terms of the Nambu spinor components) as follows. Let

$$
L\left(1,2 ; 1^{\prime}, 2^{\prime}\right)=\mathcal{G}_{2}\left(1,2 ; 1^{\prime}, 2^{\prime}\right)-\mathcal{G}\left(1,1^{\prime}\right) \mathcal{G}\left(2,2^{\prime}\right)
$$

be the two-particle correlation function, which satisfies the Bethe-Salpeter equation

$$
\begin{aligned}
L\left(1,2 ; 1^{\prime}, 2^{\prime}\right) & =-\mathcal{G}\left(1,2^{\prime}\right) \mathcal{G}\left(2,1^{\prime}\right)+\int d 3456 \mathcal{G}(1,3) \\
& \times \mathcal{G}\left(6,1^{\prime}\right) \Xi(3,5 ; 6,4) L\left(4,2 ; 5,2^{\prime}\right)
\end{aligned}
$$

where

$$
\Xi\left(1,2 ; 1^{\prime}, 2^{\prime}\right)=\frac{\delta \Sigma\left(1,1^{\prime}\right)}{\delta \mathcal{G}\left(2^{\prime}, 2\right)}
$$

is an effective two-particle interaction. Equation (6) can be formally solved in terms of the many-particle $T$ matrix, defined as the solution to the equation ${ }^{29}$

$$
\begin{aligned}
T\left(1,2 ; 1^{\prime}, 2^{\prime}\right) & =\Xi\left(1,2 ; 1^{\prime}, 2^{\prime}\right)+\int d 3456 \Xi\left(1,4 ; 1^{\prime}, 3\right) \\
& \times \mathcal{G}(3,6) \mathcal{G}(5,4) T\left(6,2 ; 5,2^{\prime}\right)
\end{aligned}
$$

by writing

$$
\begin{aligned}
-L\left(1,2 ; 1^{\prime}, 2^{\prime}\right) & =\mathcal{G}\left(1,2^{\prime}\right) \mathcal{G}\left(2,1^{\prime}\right)+\int d 3456 \mathcal{G}(1,3) \\
& \times \mathcal{G}\left(6,1^{\prime}\right) T(3,5 ; 6,4) \mathcal{G}\left(4,2^{\prime}\right) \mathcal{G}(2,5)
\end{aligned}
$$

In terms of the above quantities, one obtains the following expression for the current correlation function (1):

$$
\begin{aligned}
\chi_{\gamma, \gamma^{\prime}}\left(\mathbf{r} \tau, \mathbf{r}^{\prime} \tau^{\prime}\right) & =\frac{1}{(2 m)^{2}}\left(\nabla_{\gamma}-\nabla_{\gamma}^{\prime \prime}\right)\left(\nabla_{\gamma^{\prime}}^{\prime}-\nabla_{\gamma^{\prime}}^{\prime \prime \prime}\right) \sum_{\ell, \ell^{\prime}} \\
& \times\left. L\left(\mathbf{r}^{\prime \prime} \tau \ell, \mathbf{r}^{\prime \prime \prime} \tau^{\prime} \ell^{\prime} ; \mathbf{r} \tau^{+} \ell, \mathbf{r}^{\prime} \tau^{\prime+} \ell^{\prime}\right)\right|_{\mathbf{r}^{\prime \prime}=\mathbf{r}, \mathbf{r}^{\prime \prime \prime}=\mathbf{r}^{\prime}}
\end{aligned}
$$


In Fourier space, the above relation reads (in three dimensions):

$$
\begin{aligned}
\chi_{\gamma, \gamma^{\prime}}\left(\mathbf{q}, \Omega_{\nu}\right) & =\frac{1}{(2 m i)^{2}} \sum_{\ell, \ell^{\prime}} \int \frac{d \mathbf{p}}{(2 \pi)^{3}} \int \frac{d \mathbf{p}^{\prime}}{(2 \pi)^{3}} \frac{1}{\beta} \sum_{n} e^{i \omega_{n} \eta} \\
& \times \frac{1}{\beta} \sum_{n^{\prime}} e^{i \omega_{n^{\prime}} \eta}\left(2 p_{\gamma}+q_{\gamma}\right)\left(2 p_{\gamma^{\prime}}^{\prime}+q_{\gamma^{\prime}}\right) \\
& \times L_{\ell \ell^{\prime}}^{\ell \ell^{\prime}}\left(\mathbf{p} \omega_{n}, \mathbf{p}^{\prime} \omega_{n^{\prime}} ; \mathbf{q} \Omega_{\nu}\right)
\end{aligned}
$$

where $\mathbf{q}, \mathbf{p}$, and $\mathbf{p}^{\prime}$ are wave vectors, $\Omega_{\nu}=2 \pi \nu / \beta(\nu$ integer) is a bosonic Matsubara frequency, while $\omega_{n}=$ $(2 n+1) \pi / \beta$ and $\omega_{n}=\left(2 n^{\prime}+1\right) \pi / \beta\left(n, n^{\prime}\right.$ integers $)$ are fermionic Matsubara frequencies ( $\eta$ being a positive infinitesimal). The conventions for the (incoming and outgoing) four-vectors (with $q=\left(\mathbf{q}, \Omega_{\nu}\right), p=\left(\mathbf{p}, \omega_{n}\right)$, and $\left.p^{\prime}=\left(\mathbf{p}^{\prime}, \omega_{n^{\prime}}\right)\right)$ and for the Nambu indices are shown in Fig. 1, both for $L$ and the many-particle $T$-matrix. ${ }^{30}$

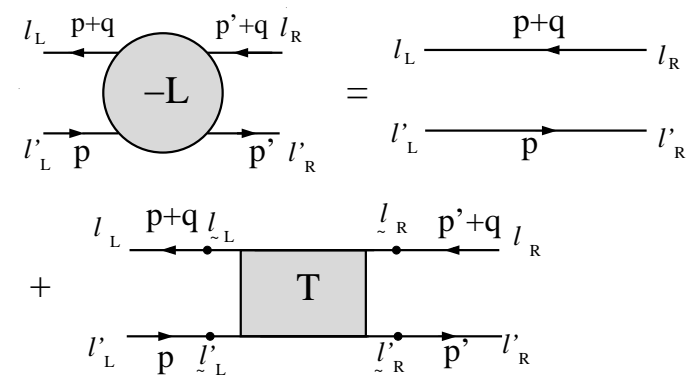

FIG. 1. Graphical representation of the two-particle correlation function $(L)$ in terms of the many-particle $T$-matrix $(T)$ (four-momenta and Nambu indices are indicated).

The above equations hold quite generally, regardless of the specific approximation for the kernel $\Xi$ defined in Eq. (7). The BCS approximation is made manifest at this point by setting:

$$
\begin{aligned}
\Xi_{B C S}\left(1,2 ; 1^{\prime}, 2^{\prime}\right) & =\frac{\delta \Sigma_{B C S}\left(1,1^{\prime}\right)}{\delta \mathcal{G}_{B C S}\left(2^{\prime}, 2\right)} \\
& =-\tau_{\ell_{1} \ell_{2^{\prime}}}^{3} \delta\left(x_{1}-x_{2^{\prime}}\right) V\left(x_{1}^{+}-x_{1^{\prime}}\right) \\
& \times \delta\left(x_{1^{\prime}}-x_{2}\right) \tau_{\ell_{1^{\prime}} \ell_{2}}^{3}\left(1-\delta_{\ell_{1} \ell_{1^{\prime}}}\right)
\end{aligned}
$$

where, as usual, only the off-diagonal terms of the BCS self-energy have been retained $\left(\tau^{3}\right.$ being a Pauli matrix, and with the additional short-hand notation $x_{1}=\left(\mathbf{r}_{1}, \tau_{1}\right)$ and so on $)$. Note that the factor $V\left(x_{1}^{+}-x_{1^{\prime}}\right)=\delta\left(\tau_{1}^{+}-\right.$ $\left.\tau_{1^{\prime}}\right) V\left(\mathbf{r}_{\mathbf{1}}-\mathbf{r}_{\mathbf{1}^{\prime}}\right)$ represents the (instantaneous) attractive effective fermionic interaction, as specified in Appendix A.

The first term on the right-hand side of Eq. (9), when inserted in Eq. (10), yields the standard BCS "bubble" contribution to the current correlation function ${ }^{1,2}$, pro- $^{-}$ vided the BCS single-particle Green's functions

$$
\begin{aligned}
& \mathcal{G}_{11}\left(\mathbf{k}, \omega_{n}\right)=-\frac{\xi(\mathbf{k})+i \omega_{n}}{E(\mathbf{k})^{2}+\omega_{n}^{2}}=-\mathcal{G}_{22}\left(-\mathbf{k},-\omega_{n}\right) \\
& \mathcal{G}_{21}\left(\mathbf{k}, \omega_{n}\right)=\frac{\Delta}{E(\mathbf{k})^{2}+\omega_{n}^{2}}=\mathcal{G}_{12}\left(\mathbf{k}, \omega_{n}\right)
\end{aligned}
$$

are used. In the above expressions, $\xi(\mathbf{k})=\mathbf{k}^{2} /(2 m)-\mu(\mu$ being the chemical potential) and $E(\mathbf{k})=\sqrt{\xi(\mathbf{k})^{2}+\Delta^{2}}$ for an isotropic ( $s$-wave) gap function $\Delta$. The BCS bubble contribution to the current correlation function taken alone does not fulfill the conservation laws (and, in particular, the longitudinal f-sum rule ${ }^{1}$ ); yet it gives a reasonable account of the transverse current correlation function in the weak-coupling limit ${ }^{1,2}$.

Quite generally, the superfluid density $\rho_{s}$ can be obtained from the knowledge of the transverse component of the current correlation function as follows ${ }^{31}$ :

$$
\sum_{\gamma, \gamma^{\prime}} \hat{t}_{\gamma} \chi_{\gamma, \gamma^{\prime}}\left(\mathbf{q} \rightarrow 0, \Omega_{\nu}=0\right) \hat{t}_{\gamma^{\prime}}=-\frac{\left(n-\rho_{s}\right)}{m}
$$

where $n$ is the density and $\hat{t}$ is a unit vector transverse to $\hat{q}=\mathbf{q} /|\mathbf{q}|$. In particular, inserting the BCS bubble contribution to the current correlation function in Eq. (14) produces a meaningful overall temperature dependence of the superfluid density in the weak-coupling limit, not only for an $s$-wave ${ }^{2}$ but also for a $d$-wave gap function $^{32,33}$. In the strong-coupling limit (and for temperatures small compared with the dissociation energy of the composite bosons), on the other hand, the BCS bubble contribution to the current correlation function vanishes, thus requiring consideration of additional diagrammatic contributions.

Still within the BCS approximation, consideration of the second term on the right-hand side of Eq. (9) is required to fulfill conservation laws, provided that BCS single-particle Green's functions (13) are consistently used $^{1}$. In particular, it can be shown that the longitudinal f-sum rule 31

$$
\sum_{\gamma, \gamma^{\prime}} \hat{q}_{\gamma} \chi_{\gamma, \gamma^{\prime}}\left(\mathbf{q} \rightarrow 0, \Omega_{\nu}=0\right) \hat{q}_{\gamma^{\prime}}=-\frac{n}{m}
$$

is fulfilled at any coupling in the static limit when this term is properly included.

It may further be of interest to obtain the full wavevector and frequency dependent current correlation function when the kernel $\Xi$ is taken within the BCS approximation. To this end, it is necessary to solve the integral equation (8) in Fourier space with the BCS form (12) of the kernel, obtained from the off-diagonal terms of the BCS self-energy. With this restriction, only four elements of the many particle $T$-matrix with $\ell_{L} \neq \ell_{L}^{\prime}$ and $\ell_{R} \neq \ell_{R}^{\prime}$ survive (cf. Fig. 1) [we adopt the convention $1 \leftrightarrow\left(\ell=1, \ell^{\prime}=2\right)$ and $2 \leftrightarrow\left(\ell=2, \ell^{\prime}=1\right)$ to label the nonvanishing matrix elements of the $T$-matrix]. In addition, when the attractive effective fermionic interaction is taken of the form of a contact potential with strength $v_{0}$ (cf. Appendix A), these four independent elements can be shown to satisfy the following algebraic equation:

$$
\begin{aligned}
& \left(\begin{array}{ll}
T_{11}(q) & T_{12}(q) \\
T_{21}(q) & T_{22}(q)
\end{array}\right)=v_{0}\left(\begin{array}{ll}
1 & 0 \\
0 & 1
\end{array}\right) \\
& +v_{0}\left(\begin{array}{cc}
-\Pi_{11}(q) & \Pi_{12}(q) \\
\Pi_{21}(q) & -\Pi_{22}(q)
\end{array}\right)\left(\begin{array}{ll}
T_{11}(q) & T_{12}(q) \\
T_{21}(q) & T_{22}(q)
\end{array}\right) .
\end{aligned}
$$


In this expression,

$$
\Pi_{11}(q)=\int \frac{d \mathbf{p}}{(2 \pi)^{3}} \frac{1}{\beta} \sum_{n} \mathcal{G}_{11}(p+q) \mathcal{G}_{11}(-p)=\Pi_{22}(-q)
$$

and

$$
\Pi_{12}(q)=\int \frac{d \mathbf{p}}{(2 \pi)^{3}} \frac{1}{\beta} \sum_{n} \mathcal{G}_{12}(p+q) \mathcal{G}_{12}(-p)=\Pi_{21}(q)
$$

are the elementary rungs on which the many-particle $T$ matrix is built up within the BCS approximation.

An equation similar to (16) holds for the particleparticle ladder in the broken-symmetry phase (cf. Appendix A). Comparison of the two equations leads us to identify:

$$
\begin{cases}T_{11}(q)=-\Gamma_{11}(q), & T_{12}(q)=\Gamma_{12}(q) \\ T_{21}(q)=\Gamma_{21}(q), & T_{22}(q)=-\Gamma_{22}(q) .\end{cases}
$$

In particular, the results obtained in Appendix $\mathrm{A}$ in the strong-coupling limit (whereby $\beta \mu \rightarrow-\infty$ ) can be exploited to write:

$$
\left\{\begin{array}{l}
T_{11}(q)=\left(8 \pi / m^{2} a_{F}\right) \mathcal{G}^{\prime}(q) \\
T_{12}(q)=\left(8 \pi / m^{2} a_{F}\right) \mathcal{G}_{21}^{\prime}(q)
\end{array}\right.
$$

where $a_{F}$ is the fermionic scattering length (which is positive in the strong-coupling limit) and $\mathcal{G}^{\prime}$ and $\mathcal{G}_{21}^{\prime}$ are the normal and anomalous noncondensate bosonic Green's functions, respectively, in the Bogoliubov approximation ${ }^{2}$.

Within the above approximations, the many-particle $T$-matrix depends only on $q$ and not on all the incoming and outgoing four-vectors (cf. Fig. 1). For this reason, the remainder (i.e., other than the bubble) contribution to the current correlation function within the BCS approximation can be cast in the following form:

$$
\begin{aligned}
\chi_{\gamma, \gamma^{\prime}}^{r e m}(q) & =I_{\gamma}(q) T_{11}(q) I_{\gamma^{\prime}}(q)+I_{\gamma}(q) T_{12}(q) I_{\gamma^{\prime}}(-q) \\
& +I_{\gamma}(-q) T_{21}(q) I_{\gamma^{\prime}}(q)+I_{\gamma}(-q) T_{22}(q) I_{\gamma^{\prime}}(-q)
\end{aligned}
$$

where we have set

$$
I_{\gamma}(q)=\frac{1}{m} \int \frac{d \mathbf{p}}{(2 \pi)^{3}} \frac{1}{\beta} \sum_{n}\left(2 p_{\gamma}+q_{\gamma}\right) \mathcal{G}_{11}(p+q) \mathcal{G}_{21}(p) .
$$

It can be readily shown that in the strong-coupling limit $I_{\gamma}(q)$ becomes

$$
I_{\gamma}(q) \simeq-q_{\gamma} \frac{m a_{F} \Delta}{16 \pi},
$$

so that the expression (21) becomes eventually with the help of Eq. (20):

$$
\chi_{\gamma, \gamma^{\prime}}^{r e m}(q) \simeq q_{\gamma} q_{\gamma^{\prime}} n_{0} \frac{1}{(2 m)^{2}}\left[\mathcal{G}^{\prime}(q)+\mathcal{G}^{\prime}(-q)-2 \mathcal{G}_{21}^{\prime}(q)\right]
$$

where the result (A37) (relating the general definition of the condensate density $n_{0}=|\alpha|^{2}$ to the off-diagonal single-particle BCS Green's function $\mathcal{G}_{12}$ in the strongcoupling limit) has been exploited. Note that, when the diagonal single-particle BCS Green's function $\mathcal{G}_{11}$ is used to evaluate the particle density $n$, a relation similar to (A37) holds for $n / 2$ in the place of $n_{0}$, implying $n_{0}=n / 2$ at the level of the above approximations. ${ }^{34}$

The expression (24) is purely longitudinal. This expression can be thus compared with the longitudinal part of the current correlation function for a system of bosons within the Bogoliubov approximation (cf. Appendix C). Identifying $n / 2$ with the bosonic density $n_{B}$ and $2 m$ with the bosonic mass $m_{B}$ (and neglecting the difference between the bosonic density $n_{B}$ and the condensate density $n_{0}$ ), one sees that the Bogoliubov result (C4) is four times smaller than the fermionic result (24) in the strongcoupling limit. This overall factor of four stems from having considered the current associated with the particle number and not with the charge density. [This is equivalent to setting formally $e=1$ for the fermionic charge and $e_{B}=2$ for the bosonic charge.] From Eq. (24) it can also be explicitly verified that the f-sum rule (15) is satisfied at this order of approximation.

Since the current correlation function for bosons within the Bogoliubov approximation contains not only a longitudinal but also a transverse part (cf. Appendix C), in the strong-coupling limit the BCS approximation for the current correlation function clearly fails to recover the expression of the current correlation function within the Bogoliubov approximation for the composite bosons. It is therefore necessary to search for additional fermionic diagrams for the current correlation function in the brokensymmetry phase, which recover the transverse Bogoliubov result in the strong-coupling limit. This task is achieved in the next Section.

\section{TRANSVERSE CURRENT CORRELATION FUNCTION IN THE BROKEN-SYMMETRY PHASE FROM THE ANALOGY WITH THE BOGOLIUBOV RESULT}

The simplest approximation for a Bose-condensed system is the so-called "Bogoliubov approximation", that relies on the system being "dilute" $35,36,2$. When one is interested in the crossover from a fermionic description of physical quantities in weak-coupling to a description in terms of (composite) bosons in strong-coupling, it is natural to require the (composite) bosons to be described by the Bogoliubov approximation, at least as a first significant step toward a more complete description of the strong-coupling limit. 
Concernig specifically the current correlation function, the form (C4) for the other-than-longitudinal contribution within the Bogoliubov approximation suggests that the fermionic diagrams to be considered should contain two particle-particle ladder propagators (of the type discussed in Appendix A). In analogy with a similar connection between fermionic and bosonic diagrams established in the normal phase $\mathrm{e}^{20}$, the Aslamazov-Larkin ${ }^{25}$ type diagram of Fig. 2(a) in the broken-symmetry phase appears to be a good candidate for our purposes. The corresponding contribution to the current correlation function reads:

$$
\begin{aligned}
\chi_{\gamma, \gamma^{\prime}}^{A L}(Q) & =-\frac{1}{(2 m)^{2}} \int \frac{d \mathbf{p}}{(2 \pi)^{3}} \frac{1}{\beta} \sum_{n} \int \frac{d \mathbf{p}^{\prime}}{(2 \pi)^{3}} \frac{1}{\beta} \sum_{n^{\prime}} \\
& \times \int \frac{d \mathbf{q}}{(2 \pi)^{3}} \frac{1}{\beta} \sum_{\nu} \sum_{\ell_{L} \ell_{L}^{\prime} \ell_{L}^{\prime \prime}} \sum_{\ell_{R} \ell_{R}^{\prime} \ell_{R}^{\prime \prime}}\left(2 p_{\gamma}+2 q_{\gamma}+Q_{\gamma}\right) \\
& \times\left(2 p_{\gamma^{\prime}}^{\prime}+2 q_{\gamma^{\prime}}+Q_{\gamma^{\prime}}\right) \Gamma_{\ell_{R}^{\prime} \ell_{L}^{\prime}}(q) \Gamma_{\ell_{L}^{\prime \prime} \ell_{R}^{\prime \prime}}(q+Q) \\
& \times \mathcal{G}_{\ell_{L} \ell_{L}^{\prime}}(p+q) \mathcal{G}_{\ell_{L}^{\prime \prime} \ell_{L}}(p+q+Q) \mathcal{G}_{\ell_{L}^{\prime \prime} \ell_{L}^{\prime}}(-p) \\
& \times \mathcal{G}_{\ell_{R}^{\prime} \ell_{R}}\left(p^{\prime}+q\right) \mathcal{G}_{\ell_{R} \ell_{R}^{\prime \prime}}\left(p^{\prime}+q+Q\right) \mathcal{G}_{\ell_{R}^{\prime} \ell_{R}^{\prime \prime}}\left(-p^{\prime}\right)
\end{aligned}
$$

where $\left(p, p^{\prime}\right)$ and $(q, Q)$ are fermionic and bosonic fourvectors, respectively, and the minus sign is due to the presence of an additional fermion loop with respect to the BCS fermionic bubble already considered in Section II. In the expression (25) we have taken into account the restrictions on the Nambu indices of the particleparticle ladder propagator, due to the regularization of the potential we have adopted. Note that all singleparticle Green's functions in Eq. (25) are meant to be taken within the BCS approximation (13).

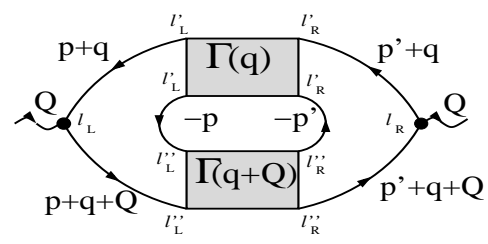

(a)

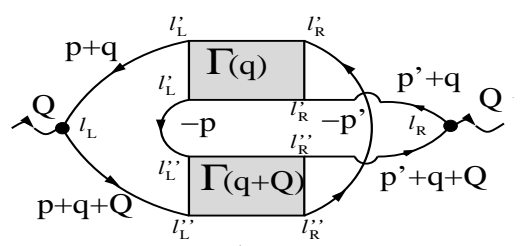

(b)

FIG. 2. (a) AL type diagram for the current correlation function in the broken-symmetry phase; (b) Diagram topologically nonequivalent to (a) but with the same value for a contact potential.

Although the expression (25) formally holds for all coupling, its calculation gets considerably simplified in the strong-coupling limit of interest, whereby the off-diagonal single-particle BCS Green's functions can be neglected with respect to the diagonal ones. In this case Eq. (25) reduces to:

$$
\begin{aligned}
\chi_{\gamma, \gamma^{\prime}}^{A L}(Q) & \simeq-\int \frac{d \mathbf{q}}{(2 \pi)^{3}} \frac{1}{\beta} \sum_{\nu} \sum_{\ell_{L} \ell_{R}} J_{\gamma}^{\ell_{L}}(q, Q) \Gamma_{\ell_{R} \ell_{L}}(q) \\
& \times \Gamma_{\ell_{L} \ell_{R}}(q+Q) J_{\gamma^{\prime}}^{\ell_{R}}(q, Q)
\end{aligned}
$$

where we have introduced the vertex

$$
\begin{aligned}
J_{\gamma}^{\ell}(q, Q) & =\frac{1}{2 m} \int \frac{d \mathbf{p}}{(2 \pi)^{3}} \frac{1}{\beta} \sum_{n}\left(2 p_{\gamma}+2 q_{\gamma}+Q_{\gamma}\right) \\
& \times \mathcal{G}_{\ell \ell}(p+q) \mathcal{G}_{\ell \ell}(p+q+Q) \mathcal{G}_{\ell \ell}(-p) .
\end{aligned}
$$

Since $J_{\gamma}^{2}(q, Q)=J_{\gamma}^{1}(-q,-Q)$ owing to the symmetry relations in Eq. (13), it is sufficient to evaluate $J_{\gamma}^{1}(q, Q)$. One obtains:

$$
J_{\gamma}^{1}(q, Q) \simeq-\frac{\left(2 q_{\gamma}+Q_{\gamma}\right)}{2 m} \frac{m^{2} a_{F}}{16 \pi}
$$

in agreement with the value obtained for the corresponding vertex of the AL diagram in the normal phase ${ }^{20}$. Making explicit the Nambu indices in Eq. (26) and recalling Eqs. (19) and (20) for the connection between the elements of the particle-particle ladder and the bosonic propagators within the Bogoliubov approximation, we obtain eventually in the strong-coupling limit:

$$
\begin{aligned}
\chi_{\gamma, \gamma^{\prime}}^{A L}(Q) & \simeq-\frac{1}{(2 m)^{2}} \frac{1}{4} \int \frac{d \mathbf{q}}{(2 \pi)^{3}} \frac{1}{\beta} \sum_{\nu}\left(2 q_{\gamma}+Q_{\gamma}\right) \\
& \times\left(2 q_{\gamma^{\prime}}+Q_{\gamma^{\prime}}\right)\left[\mathcal{G}^{\prime}(q) \mathcal{G}^{\prime}(q+Q)+\mathcal{G}^{\prime}(-q)\right. \\
& \left.\times \mathcal{G}^{\prime}(-q-Q)-2 \mathcal{G}_{21}^{\prime}(q) \mathcal{G}_{21}^{\prime}(q+Q)\right] .
\end{aligned}
$$

This expression has to be compared with the current correlation function for bosons within the Bogoliubov approximation, i.e., with the second term on the right-hand side of Eq. (C4). The comparison shows that expression (29) is only two times larger than its Bogoliubov counterpart, while one would expect it to be four times larger according to the discussion following Eq. (24). The missing factor of two is provided by an additional diagram [cf. Fig. 2(b)], which is topologically nonequivalent to the diagram of Fig. 2(a) but has the same value for a contact potential.

In the weak-coupling limit, on the other hand, the AL type diagrams of Fig. 2 in the broken-symmetry phase represent fluctuation contributions to the ordinary BCS bubble approximation, and merge with the usual Aslamazov-Larkin contribution ${ }^{25}$ when approaching the critical temperature from below.

Having succeeded in obtaining the Bogoliubov result for the transverse part of the current correlation function in the broken-symmetry phase as the strong-coupling limit of the fermionic diagrams of Fig. 2, we pass now to determine the evolution of the transverse part of the current correlation function from weak to strong coupling, by approximating the current correlation function in all 
coupling regimes as the sum of the standard BCS bubble diagram and the diagrams of Fig. 2. In particular, we shall be interested in the static limit of these diagrams, from which the superfluid density $\rho_{s}$ can be extracted as $\mathbf{q} \rightarrow 0$ according to Eq. (14) and the Pippard kernel can be obtained by retaining finite values of $\mathbf{q}$. The numerical results obtained, from weak to strong coupling, for the superfluid density as a function of temperature and for the Pippard kernel at zero temperature are discussed in the next Section.

\section{NUMERICAL RESULTS FOR THE SUPERFLUID DENSITY AND THE PIPPARD-LIKE KERNEL FROM WEAK TO STRONG COUPLING}

In this Section, we evaluate numerically the AL type contribution (25) to the current correlation function for vanishing external frequency from weak to strong coupling, in addition to the standard BCS bubble contribution. From this calculation we extract the temperature dependence of the superfluid density (when the external wave vector is also allowed to vanish) as well as the form of the Pippard-like kernel at zero temperature (by keeping the external wave vector finite).

While the numerical calculation of the BCS bubble is rather straightforward, calculation of the expression (25) requires a non-negligible numerical effort. Specifically, evaluation of the particle-particle ladder $\Gamma$ [cf. Eqs. (A9) and (A10)], where the frequency sum can be done analytically, requires a two-dimensional numerical integration; in addition, the four independent vertices with three single-particle Green's functions (of which the expression (27) represents a degenerate case), where again the frequency sum can be done analytically, require a two-dimensional (three-dimensional) numerical integration for vanishing (finite) external wave vector; finally, both the frequency sum and the wave vector integration over the bosonic variable $q$ of the $\Gamma$ functions have to be done numerically. Special care has to be exerted when performing this frequency sum, which requires explicit inclusion of about 300 to 500 (positive) Matsubara frequencies, as well as a careful treatment of the tail of the frequency sum which we approximate by an integral extending up to infinity. The resulting function (to be eventually integrated over the wave vector $\mathbf{q}$ ) is, in fact, affected by spurious oscillations for large values of $|\mathbf{q}|$, which result from incomplete cancellations of large numbers when the tail of the above frequency sum is added to the contribution of the finite set of Matsubara frequencies. In addition, these oscillations are strongly amplified by the factor $\mathbf{q}^{4}$ originating from the measure of the (three-dimensional) integral and from the wave-vector dependence of the vertices with three Green's functions. As a consequence, we have pragmatically truncated the integral over $|\mathbf{q}|$ where these spurious oscillations start to appear. We have consistently verified that this truncation procedure yields correct results in the two cases where analytic controls can be made, namely, in the strong-coupling limit at low enough temperatures and in the weak-coupling limit close to the (BCS) critical temperature.

A comment on the procedure we have adopted to generate the values of the gap function $\Delta$ and chemical potential $\mu$ is in order at this point (these parameters enter the single-particle Green's functions (13) and, through them, all other relevant quantities). According to a standard procedure when following the evolution from BCS superconductivity to $\mathrm{BE}$ condensation ${ }^{13,14}, \Delta$ and $\mu$ are obtained by solving the coupled equations for the meanfield gap and particle number, in terms of the Green's functions (13). Strictly speaking, these equations are valid at low enough temperature (compared with the superconducting critical temperature), and corrections to these equations should accordingly be considered at higher temperatures. In this paper, we restrict to low enough temperatures, so that inclusion of these corrections is not expected to be important. Improved results at higher temperatures may then be obtained by supplying the present calculation for the current correlation function with improved values of $\Delta$ and $\mu$, which take into account the effects of superconducting fluctuations below $T_{c} \cdot 34$

\section{A. Superfluid density}

Once the AL type and the fermionic bubble contributions to the current correlation function are calculated as described above, the temperature dependence of the superfluid density $\rho_{s}$ can be obtained via the definition (14) by setting $\Omega_{\nu}=0$ and letting $\mathbf{q} \rightarrow 0$.

The low-temperature behavior of the fermionic bubble leads to the well-known exponential suppression of the normal density $n-\rho_{s}$ when approaching zero temperature; this exponential behavior is governed by the BCS gap in weak coupling and by the magnitude of the chemical potential in strong coupling. These energy scales, in turn, limit the respective temperature range for the validity of the exponential behavior. The low-temperature behavior of the AL type diagram in the strong-coupling limit, on the other hand, yields a $T^{4}$ increase of the normal density for increasing temperature, in agreement with the Bogoliubov-Landau expression ${ }^{2}$ (cf. Appendix C). In this limit, the $T^{4}$ behavior is valid for temperatures small compared with the bosonic chemical potential and is due to the linear dispersion of the Bogoliubov mode for small enough wave vectors. Since the poles of the particle-particle ladder $\Gamma$ yield a linear dispersion even at intermediate and weak coupling ${ }^{37}$ (giving origin to the so-called Bogoliubov-Anderson mode ${ }^{38}$ ), one expects the $T^{4}$ behavior from the AL type contribution to survive at low enough temperature for all coupling ranges. This $T^{4}$ 
behavior is thus expected to dominate over the BCS exponential behavior at low enough temperatures. [Note, however, that the $T^{4}$ behavior is bound to cross over into a different power-law behavior at a finite characteristic temperature, as discussed below.]

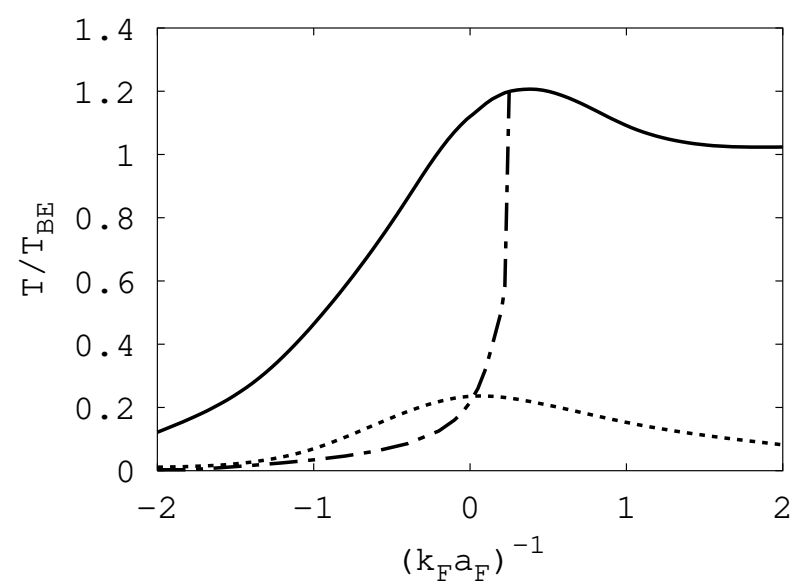

FIG. 3. Temperatures below which (dashed-dotted line) the AL type contribution to the normal density is dominant with respect to the BCS contribution and below which (dotted line) the $T^{4}$ behavior of the AL type contribution sets in, versus the coupling parameter $\left(k_{F} a_{F}\right)^{-1}$. The superconducting critical temperature $T_{c}$ (full line) is also shown for comparison. [All temperatures are in units of the Bose-Einstein condensation temperature $T_{B E}=3.31 n_{B}^{2 / 3} / m_{B}$.]

An interesting question that can be answered with the present calculation is to determine the temperature range below which the $\mathrm{AL}$ type contribution dominates over the BCS contribution on the whole coupling range, from weak to strong. To this end, we have calculated the temperature at which these two contributions equal each other for several values of the coupling parameter $\left(k_{F} a_{F}\right)^{-1}$ (where $k_{F}$ is the Fermi wave vector and $a_{F}$ is the scattering length - cf. Appendix A). [In practice, the whole crossover region can be spanned by varying $\left(k_{F} a_{F}\right)^{-1}$ about from -1 to +1 ; deviations from weakcoupling approximations when $\left(k_{F} a_{F}\right)^{-1} \lesssim-1$ and from strong-coupling approximations when $\left(k_{F} a_{F}\right)^{-1} \gtrsim 1$ become, in fact, negligible for all practical purposes.] In Fig. 3 this temperature is plotted versus $\left(k_{F} a_{F}\right)^{-1}$, together with the superconducting critical temperature $T_{c}$ obtained following Ref. 15 (cf. also Refs. 16 and 17). In the same plot, we have also reported the temperature at which the AL type contribution changes from the $T^{4}$ behavior to a different power law, from which one notes that the range of validity of the "low-temperature" behavior is significantly enhanced in the crossover region. [It is, however, clear from the previous discussion that the results for these two boundary temperatures are only indicative when the two temperatures become of order $T_{c}$.] Note also that the region where the AL type contribution dominates over the BCS bubble is resticted to very low temperatures in weak coupling, while it increases rapidly in the crossover region. In particular, it reaches about one-fifth of the critical temperature at $\left(k_{F} a_{F}\right)^{-1}=0$, a coupling value for which the chemical potential is still positive and remnants of the Fermi surface survive (the chemical potential changing sign at $\left.\left(k_{F} a_{F}\right)^{-1}=0.55\right)$. The inclusion of the AL type diagram turns thus out to be appreciable as soon as one leaves the extreme weak-coupling region. In the strong-coupling side of the crossover region (i.e., for $\left.\left(k_{F} a_{F}\right)^{-1} \gtrsim 0.3\right)$, the AL type contribution definitely dominates over the BCS contribution for all relevant temperatures.
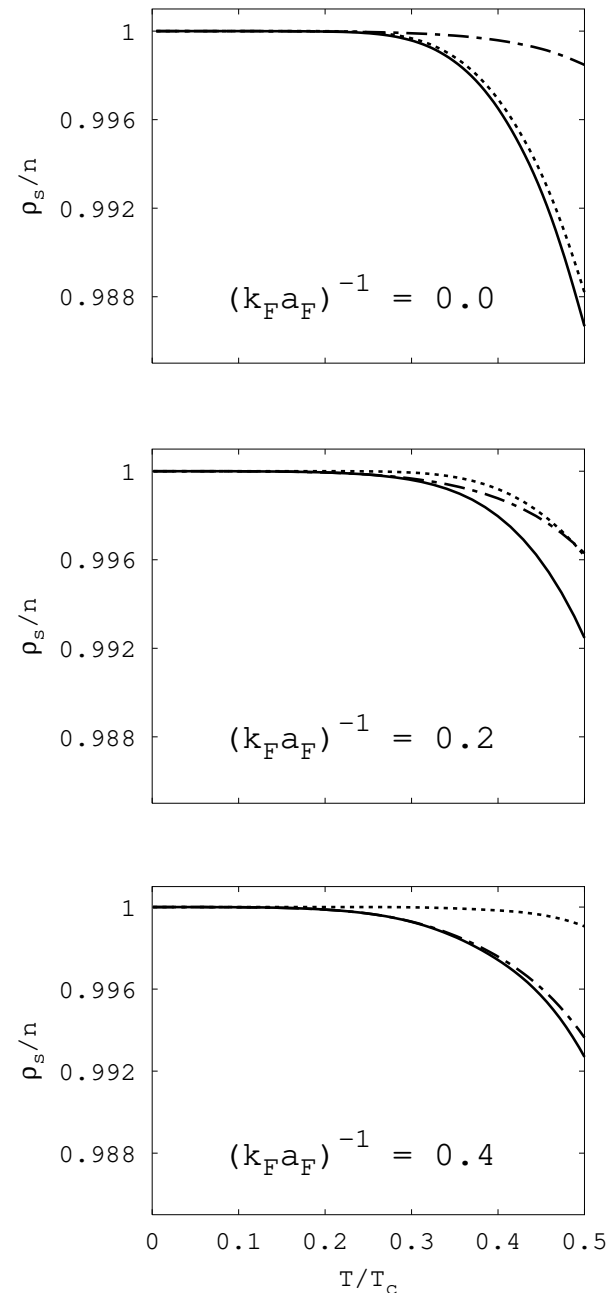

FIG. 4. Temperature dependence of the AL type (dashed-dotted line) and BCS (dotted line) contributions to the superfluid density, and sum of the two contributions (full line), vs temperature when $\left(k_{F} a_{F}\right)^{-1}=0.0,0.2,0.4$, from top to bottom. [The temperature is in units of $T_{c}$ as obtained from Fig. 3.]

The temperature dependence of the AL type and BCS contributions to the superfluid density is shown in Fig. 4 for three characteristic couplings in the crossover region, 
namely, $\left(k_{F} a_{F}\right)^{-1}=0.0,0.2,0.4$. We have extended this calculation up to halfway the critical temperature, where the values of $\Delta$ and $\mu$ obtained as described above are still reliable. Note that for $\left(k_{F} a_{F}\right)^{-1}=0.0$ the BCS contribution dominates over the AL type contribution, while for $\left(k_{F} a_{F}\right)^{-1}=0.4$ the opposite occurs. This implies that, even for the superfluid density, the crossover from weak- to strong-coupling behavior occurs in a rather narrow region of the coupling parameter $\left(k_{F} a_{F}\right)^{-1}$. The mechanism for this rapid crossover is that the BCS contribution becomes quickly negligible when $\left(k_{F} a_{F}\right)^{-1}$ approaches zero from negative values $\left(\left(k_{F} a_{F}\right)^{-1}=0\right.$ corresponds to the threshold for the occurrence of a bound state in the associated two-body problem); the AL type contribution, on the other hand, becomes quickly negligible when $\left(k_{F} a_{F}\right)^{-1}$ approaches zero from positive values. It turns thus out that there exists a finite interval of the parameter $\left(k_{F} a_{F}\right)^{-1}$ where both contributions are small with respect to the full density $n$ (at fixed temperature). From a physical point of view, one intepretes this result by noting that there are two possible mechanisms to destroy the superfluid density, namely, the pair-breaking effect on the weak-coupling side and the excitation of collective modes on the strong-coupling side. As both mechanisms tend to be suppressed in the intermediatecoupling region, the value of the superfluid density is correspondingly enhanced and superfluidity gets more robust against thermal fluctuations.

To establish comparison with the experimental data on cuprate superconductors, the calculation of the temperature dependence of the superfluid density should realistically include also lattice effects and the $d$-wave character of the gap function. Nevertheless, we expect the above physical argument (about the increasing robustness of the superfluid density against thermal fluctuations when approaching the intermediate-coupling region from both sides) to remain valid even in the presence of a $d$-wave gap function. In fact, in the presence of a $d$-wave gap function the BCS contribution gets suppressed even faster for increasing coupling as soon as the chemical potential crosses the bottom of the single-particle energy band, passing from a linear- $T$ behavior ${ }^{32,33}$ to an ( $s$-wave like) exponential behavior; the AL type contribution in the presence of a $d$-wave gap, on the other hand, should not be appreciably modified with respect to the results obtained with an $s$-wave gap, at least when the chemical potential remains below the bottom of the single-particle band.

An example of a superconductor with an $s$-wave gap function, for which the effective coupling may be sufficiently strong that the effect of collective modes on the superfluid density could be revealed, is the recently discovered $\mathrm{MgB}_{2}$. Experimental data for the temperature dependence of the penetration depth (and thus of the superfluid density) show a quadratic temperature dependence over most of the temperature range up to $T_{c}$, but for noticeable deviations in the low-temperature region which appear instead to follow a quartic behavior ${ }^{39}$.
While the quadratic temperature dependence away from the low-temperature region may simply result from an ordinary ( $s$-wave) BCS contribution (which can indeed be rather well fitted to a quadratic behavior upon decreasing the temperature from $T_{c}$ ), the quartic behavior at low temperature may instead be due to the presence of collective modes. [The occurrence of the above quadratic temperature dependence at high enough temperatures has alternatively be considered as an indication of the presence of nodes in the superconducting energy gap of $\left.M g B_{2} \cdot{ }^{39}\right]$ In Fig. 5 we report the superfluid density versus $T^{2}$, as obtained by our calculations with both BCS and AL type contributions included, for a specially chosen value of the coupling $\left(\left(k_{F} a_{F}\right)^{-1}=-0.4\right)$ such that both the $T^{4}$ behavior (due to the AL type contribution at low temperatures) and the $T^{2}$ behavior (due to the BCS contribution at higher temperatures) are evident. Note that, to evidence the $T^{2}$ behavior at high enough temperatures, the numerical results reported in Fig. 5 have been extended beyond their strict range of validity, where the values of $\Delta$ and $\mu$ should rather be obtained by an improved calculation as mentioned above. For this reason, the temperature scale in Fig. 5 has been normalized with respect to the mean-field critical temperature $T_{\mathrm{MF}}$ at which $\Delta$ vanishes in the present calculation (and not with respect to $T_{c}$ reported in Fig. 3 ). Note also that, while the experimental curve reported in Ref. 39 shows a change of convexity when passing from the $T^{2}$ to the $T^{4}$ behavior, the theoretical curve shown in Fig. 5 mantains the same convexity over the whole temperature range.

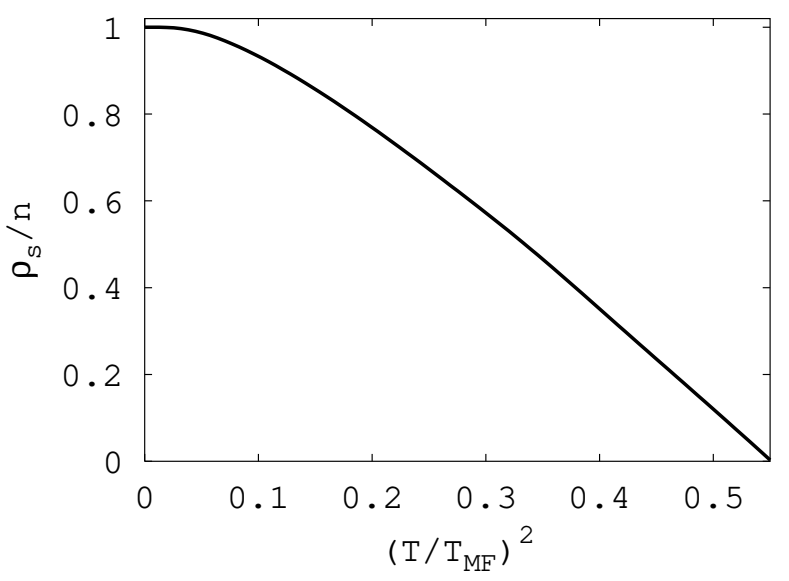

FIG. 5. Superfluid density vs $T^{2}$ when $\left(k_{F} a_{F}\right)^{-1}=-0.4$, including both BCS and AL type contributions. [The temperature is in units of the mean-field critical temperature.]

Care should definitely be exerted when trying to compare the temperature dependence of the superfluid density, obtained also with the inclusion of collective modes, with the experimental data for superconductors where density fluctuations produce charge unbalance. In this case, the Bogoliubov mode with a linear dispersion at 
small wave vectors should turn into a gapped plasmon mode owing to the long-range Coulomb interaction. Dissipation effects have also been recently shown to play an important role in restoring a power-law behavior for the superfluid density at low temperature, albeit with a different exponent from that obtained by considering only neutral collective modes ${ }^{40}$. Renormalization of the chemical potential (which drives the system from the BCS to the BE limit) has, however, not been considered in this context. A more realistic approach to the calculation of the low-temperature behavior of the superfluid density would then include both Coulomb and damping effects on top of the treatment presented in this paper, which is appropriate to follow the crossover from the BCS to the BE limit. Assessment of the relative importance of quasi-particle excitations and collective modes to the superfluid density, in fact, can be realistically done only in the context of this crossover when the chemical potential is allowed to be properly renormalized. This more complete approach is deferred to future work.

Notwithstanding these limitations, it is interesting to exploit further our calculation for the current correlation at zero frequency, to obtain the form of the Pippard-like kernel from weak to strong coupling at zero temperature.

\section{B. Pippard-like kernel}

As discussed in the Introduction, two different length scales $\xi_{\text {phase }}$ (describing correlation among different Cooper pairs or composite bosons) and $\xi_{\text {pair }}$ (describing correlation between fermions in a pair) enter the BCS$\mathrm{BE}$ crossover problem. In particular, while $\xi_{\text {phase }} \sim \xi_{\text {pair }}$ in the weak-coupling limit, $\xi_{\text {phase }} \gg \xi_{\text {pair }}$ in the strongcoupling limit. ${ }^{6}$ In addition, in the weak-coupling limit $\xi_{\text {pair }}$ coincides with the Pippard coherence length $\xi_{0}$ characterizing the non-local relation between the supercurrent $\mathbf{j}$ and the vector potential $\mathbf{A} .{ }^{26}$ It is then natural to ask which one of the two lengths ( $\xi_{\text {pair }}$ or $\left.\xi_{\text {phase }}\right)$ enters this relation between the supercurrent and the vector potential also in the intermediate- and strong-coupling regions. In this subsection, we consider the contribution to the $\mathbf{j}$ vs $\mathbf{A}$ relation from the standard BCS bubble and the AL type diagram introduced in Section III, and show that $\xi_{\text {pair }}$ and $\xi_{\text {phase }}$ are associated with the two contributions, in the order.

Quite generally, to extract the Pippard-like kernel (whose precise definition will be given below) from the expression of the current correlation function in a form appropriate to numerical calculations, it is convenient to identify from Eq. (25) the function $G^{A L}(\mathbf{q}, \mathbf{p})$, such that

$$
\begin{aligned}
\chi_{\gamma, \gamma^{\prime}}^{A L}\left(\mathbf{Q}, \Omega_{\nu}=0\right) & =-\frac{1}{(2 m)^{2}} \int \frac{d \mathbf{q}}{(2 \pi)^{3}}\left(2 q_{\gamma}+Q_{\gamma}\right) \\
& \times\left(2 q_{\gamma^{\prime}}+Q_{\gamma^{\prime}}\right) \frac{G^{A L}(\mathbf{q}, \mathbf{q}+\mathbf{Q})}{2|\mathbf{q}||\mathbf{q}+\mathbf{Q}|}
\end{aligned}
$$

for the transverse components (we shall restrict, in particular, to the zero-temperature limit). With this definition, the function $G^{A L}(\mathbf{q}, \mathbf{p})$ reduces to the expression (C7) of Appendix $\mathrm{C}$ in the strong-coupling limit.

For all couplings, it turns out that $G^{A L}(\mathbf{q}, \mathbf{p})=$ $G^{A L}(|\mathbf{q}|,|\mathbf{p}|)$ and that $G^{A L}(q, q)=0$, as in the Bogoliubov case (cf. Appendix C). [We have verified these properties numerically for the AL case within a $5 \%$ accuracy.] In this way, Eqs. (C9)-(C12) of Appendix $\mathrm{C}$ remain formally valid for all couplings (with the label AL attached to the quantities $G(p, q)$ and $g(R)$ therein).

Strictly speaking, however, it is no longer possible to extract the delta-function from the $\mathrm{AL}$ contribution at arbitrary coupling as in Eq. (C13), since for composite bosons $\lim _{p \rightarrow \infty} p G^{A L}(q, p)=0$ (instead of the behavior (C15) for point-like bosons). For composite bosons the coefficient $\mathcal{I}$ of Eq. (C13) vanishes and only the nonlocal contribution $g_{n}^{A L}(R)$ survives. Nonetheless, upon approaching the extreme strong-coupling limit (where the composite nature of the bosons no longer matters) the small- $R$ behavior of $g_{n}^{A L}(R)$ can be effectively assimilated to a delta-function (since its range extends over a length scale much smaller than the scale $\xi_{\text {phase }}$ associated with the remaining part of $g_{n}^{A L}(R)$ ), such that the expected behavior for point-like bosons is properly recovered in this limit, as also shown below by the numerical results.

Similarly, the BCS bubble contribution can be calculated at arbitrary couplings according to the procedure of Appendix D, instead of relying on the standard BCS analytic calculation for weak-coupling ${ }^{2}$. In this case, one can identify the function $g_{n}^{\mathrm{BCS}}(R)$ for $R \neq 0$ and its integral $\mathcal{I}^{\mathrm{BCS}}=-8 \pi^{3} m n$ (cf. Eq. (D11)). What happens to the BCS bubble contribution is that, in the weakcoupling limit, the function $g_{n}^{\mathrm{BCS}}(R)$ contains a shortrange part extending over the scale $k_{F}^{-1} \ll \xi_{\text {pair }}$, whose integral (from $R=0$ to $R=k_{F}^{-1}$ ) together with the deltafunction contribution proportional to $\mathcal{I}^{\mathrm{BCS}}$ cancels the diamagnetic contribution to the $\mathbf{j}$ vs $\mathbf{A}$ relation, making only the nonlocal part of $g_{n}^{\mathrm{BCS}}(R)$ effectively surviving.

Putting together the results for the AL and BCS contributions, the desired relation between the induced current $\mathbf{j}$ and the vector potential $\mathbf{A}$ acquires the form:

$$
\begin{aligned}
j_{\gamma}(\mathbf{r}) & =-\frac{n}{3 m c} A_{\gamma}(\mathbf{r}) \\
& +\frac{1}{(2 \pi)^{4} m^{2} c} \int d \mathbf{r}^{\prime} \frac{R_{\gamma} \sum_{\gamma^{\prime}} R_{\gamma^{\prime}} A_{\gamma^{\prime}}\left(\mathbf{r}^{\prime}\right)}{R^{4}} \\
& \times\left(g_{n}^{B C S}(R)+g_{n}^{A L}(R)\right)
\end{aligned}
$$

where $\mathbf{R}=\mathbf{r}-\mathbf{r}^{\prime}$ and $c$ is the light velocity. This relation defines the Pippard-like kernel at arbitrary couplings, which is the sum of a local (London-like) and of a nonlocal component. Note that the local part of Eq. (31) coincides with the local part of Eq. (D12) since the AL contribution lacks the delta function.

The results of the numerical calculation for the two (BCS and AL) contributions to Eq. (31) show that, in the weak-coupling limit, the BCS contribution dominates 
over the AL counterpart even though both contributions extend over the same spatial range since $\xi_{\text {pair }} \sim \xi_{\text {phase }}$ in this limit. In the strong-coupling limit, on the other hand, the BCS bubble no longer contributes significantly to the response function since the nonlocal part $g_{n}^{B C S}(R)$ cancels the delta-function contribution over a scale $\xi_{\text {pair }}$ much smaller than the scale $\xi_{\text {phase }}$ of the AL contribution. At arbitrary coupling, both BCS and AL contributions to Eq. (31) need be explicitly considered.

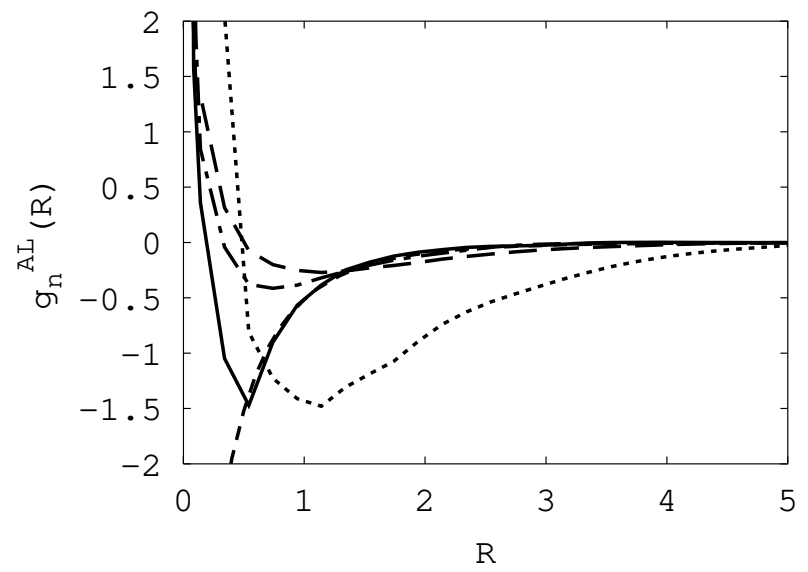

FIG. 6. Function $g_{n}^{A L}(R)$ (in units of $\left.m_{B} /\left(4 \xi_{\text {phase }}^{4}\right)\right)$ vs $R$ (in units of $\sqrt{2} \xi_{\text {phase }}$ ) for four different values of the coupling parameter $\left(k_{F} a_{F}\right)^{-1}$ : -1.0 (dotted line), 0.0 (long-dashed line), +1.0 (dashed-dotted line), +2.0 (full line). The purely bosonic $g_{n}^{B}(R)$ (short-dashed line) is also shown for comparison.

In Fig. 6 the functions $g_{n}^{A L}(\tilde{R})$ is reported vs $\tilde{R}=$ $R /\left(\sqrt{2} \xi_{\text {phase }}\right)$ for different values of the coupling parameter $\left(k_{F} a_{F}\right)^{-1}$. The relevant range of $g_{n}^{A L}(\tilde{R})$ extracted from this plot is determined by the coherence length $\xi_{\text {phase }}$ introduced in Ref. 6 to account for the spatial correlations of the amplitude of the order parameter (direct calculation ${ }^{37}$ of $k_{F} \xi_{\text {phase }}$ yields the values $(1.7,0.7,0.8,1.1)$ corresponding to the values $\left(k_{F} a_{F}\right)^{-1}=(-1.0,0.0,+1.0,+2.0)$ here considered). [We have also verified that $k_{F} \xi_{\text {phase }}$ extracted from the behavior of $g_{n}^{A L}(R)$ attains its minimum at $\left(k_{F} a_{F}\right)^{-1}=$ +0.4 , in agreement with the results from Ref. 6.] Note that the delta-function contribution to $g(R)$, which is present in the bosonic expression of Appendix $\mathrm{C}$, is recovered in Fig. 6 by the progressive shrinking of the short range part of $g_{n}^{A L}(R)$ as the coupling is increased; at the same time, the long-range part of $g_{n}^{A L}(R)$ in Fig. 6 approaches the asymptotic behavior of the bosonic $g_{n}^{B}(R)$. We should warn, however, that extracting the shortrange part of $g_{n}^{A L}(R)$ from numerical calculation of the AL type diagram is affected by sizable numerical errors, since it requires an accurate treatment of the large wavevector behavior of $G^{A L}(q, p)$ (this procedure, in turn, requires subtraction of large terms which should add up to zero).

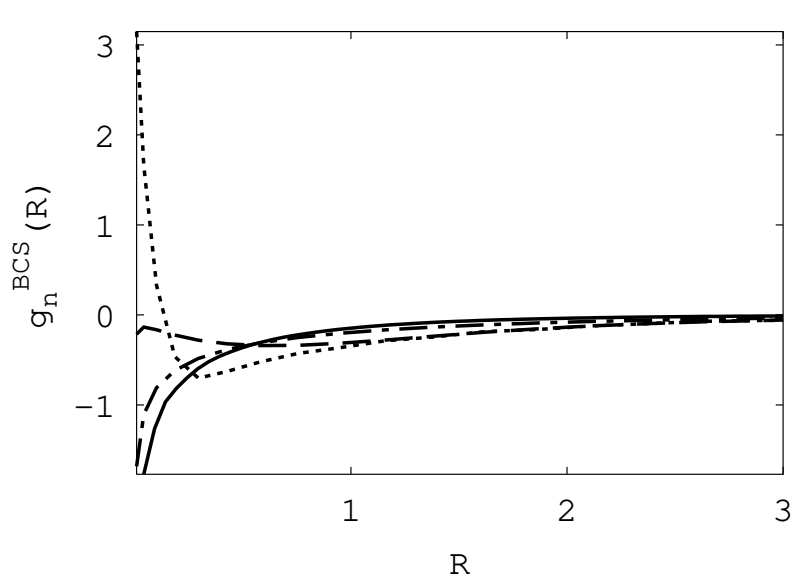

FIG. 7. Function $g_{n}^{B C S}(R)$ (in units of $m k_{F}^{4}$ ) vs $R$ (in units of $\left.\xi_{\text {pair }}\right)$ for four different values of the coupling parameter $\left(k_{F} a_{F}\right)^{-1}:-2.0$ (dotted line), -1.0 (dashed line), 0.0 (dashed-dotted line), +1.0 (full line).

In Fig. 7 the function $g_{n}^{B C S}(\tilde{R})$ is reported vs $\tilde{R}=$ $R / \xi_{\text {pair }}$ for different values of the coupling parameter $\left(k_{F} a_{F}\right)^{-1}$. The relevant range of $g_{n}^{B C S}(\tilde{R})$ is determined by the coherence length $\xi_{\text {pair }}$ for two-fermion correlation $^{5,6}$, which is monotonically decreasing for increasing coupling. In particular, direct calculation ${ }^{37}$ of $k_{F} \xi_{\text {pair }}$ yields the values $(15.2,3.4 .1 .1,0.6)$ for $\left(k_{F} a_{F}\right)^{-1}=(-2.0,-1.0,0.0,+1.0)$, respectively, in agreement with the values extracted from Fig. 7 .

We recall at this point that (apart from a numerical factor of order unity) $\xi_{\text {phase }}$ and $\xi_{\text {pair }}$ coincide with each other in the intermediate-to-weak-coupling side of the crossover (which is mostly relevant to the BCS contribution). We thus conclude from the present analysis of the current correlation function that it is the length $\xi_{\text {phase }}$ to determine the range of the Pippard-like kernel over the whole crossover range. In addition, the present discussion shows the importance of including both BCS and AL type contributions in the intermediate-coupling region, for a correct description of the current correlation function.

\section{CONCLUDING REMARKS}

In this paper, we have calculated the current correlation function within the BCS-BE crossover. We have complemented the standard analysis of the BCS diagrammatic contribution to the current correlation function in the broken-symmetry phase, by considering an additional (Aslamazov-Larkin type) diagrammatic contribution which includes fluctuation effects in weak coupling and recovers the Bogoliubov form of the current correlation function in strong coupling.

In view of application to high-temperature cuprate superconductors, the analysis presented in this paper is 
necessarily a preliminary one, since it relies on a continuum model in three dimensions and does not take into account additional effects (such as the Coulomb repulsion between charged composite bosons), which modify the Bogoliubov picture for bosons. In addition, lattice effects and the $d$-wave character of the gap function should also be included in a realistic calculation of the superfluid density.

The analysis presented in this paper is, nonetheless, a required preliminary step toward a more realistic theory of the current correlation function in the superconducting phase, by connecting results valid in the weak- and strong-coupling limits in the spirit of the BCS-BE crossover. In this context, the identification of the Aslamazov-Larkin type diagram(s) in the brokensymmetry phase as the dominant one(s) in the strongcoupling limit of the theory represents one of the main contributions of the present paper. In addition, consideration of the evolution of the current correlation function in the broken-symmetry phase from weak to strong coupling has required us to deal with several relevant theoretical issues, such as the mapping between the fermionic and bosonic diagrammatic structures in the broken-symmetry phase, the connection between the Bogoliubov propagators for composite bosons and the diagrammatic structure for the constituent fermions, and the identification of the local and nonlocal parts of the $\mathbf{j}$ vs $\mathbf{A}$ relation for a generic diagrammatic contribution to the current correlation function. The temperature dependence of the superfluid density that we have obtained (by including the AL type diagram on top of the conventional BCS "bubble" diagram with an $s$-wave gap function) has served to identify the temperature regions where one of the two contributions dominates over the other one in different coupling regimes. In this context, we have shown that, in the intermediate-coupling region, superfluidity is most robust against suppressing mechanisms, such as pair breaking (coming from the weak-coupling side) and collective excitations (coming from the strong-coupling side), since the effects of these mechanisms is considerably reduced in the intermediate-coupling region. It would accordingly be tempting to relate this effect to the occurrence of a maximum for the superconducting critical temperature in the phase diagram of cuprate superconductors at intermediate doping. We have also been able to extract from our (zero-temperature) calculation of the spatial dependence of the current response kernel a length scale previously introduced in the theory of the BCS-BE crossover, which specifies the correlation among different fermion pairs.

Although detailed comparison with experimental data on cuprate superconductors has to await more realistic calculations that include lattice effects and the $d$-wave character of the gap function (for instance, via an extended negative- $U$ Hubbard model), the trend of the coherence length in the Pippard-like kernel to decrease initially with increasing coupling from weak to intermediate should be experimentally accessible for cuprate supercon- ductors upon decreasing the doping (from overdoping to underdoping), in analogy with the original experimental results by Pippard ${ }^{26}$.

Previous calculations of the temperature dependence of the superfluid density have included the $d$-wave character of the gap function within the standard BCS "bubble" contribution $^{33}$, or have considered incoherent pair excitations of finite momentum which assist the destruction of superconductivity (still taking into account the $d$-wave gap) in the context of the BCS-BE crossover ${ }^{41}$. As both works do not address the role of collective modes (over and above the quasi-particle contribution) in the context of this crossover, direct comparison with our work cannot be made. Additional inclusion of long-range Coulomb interaction addressed in Ref. 40 is more directly relevant to the present approach and is presently being considered.

\section{ACKNOWLEDGMENTS}

The authors are indebted to C. Castellani for discussions. Financial support from the Italian MIUR under contract COFIN 2001, Prot. 2001023848 is gratefully acknowledged.

\section{APPENDIX A: PARTICLE-PARTICLE LADDER PROPAGATOR IN THE BROKEN-SYMMETRY PHASE AND THE BOGOLIUBOV PROPAGATORS}

In this Appendix, we study in detail the particleparticle ladder entering the fermionic diagrammatic structure for the broken-symmetry phase, and specifically the AL diagram(s) considered in Section III. To this end, we shall take the fermionic attractive interaction of the form of a contact-potential with a suitable regularization, as it was done in Ref. 9 for the normal phase. In addition, in the strong-coupling limit we establish a connection between the particle-particle ladder propagator and the Bogoliubov propagators for bosons, correcting at the same time some erroneous conclusions reached in Ref. 4 in this context.

Knowledge of the detailed form of the attractive interaction $V(\mathbf{r})$ of Eq. (12) is not required when studying the BCS-BE crossover. One may accordingly consider the simple form of a "contact" potential $V(\mathbf{r})=v_{0} \delta(\mathbf{r})$, where $v_{0}$ is a negative constant. This choice of the potential entails a suitable regularization, e.g., in terms of a cutoff $k_{0}$ in wave-vector space. In three dimensions, this can be achieved via the scattering length $a_{F}$ of the associated two-body (fermionic) problem, by choosing the constant $v_{0}$ as follows ${ }^{9}$ :

$$
v_{0}=-\frac{2 \pi^{2}}{m k_{0}}-\frac{\pi^{3}}{m a_{F} k_{0}^{2}} .
$$


In this way, the classification of the (fermionic) manybody diagrams is considerably simplified even in the broken-symmetry phase, since only specific diagrammatic substructures survive when the limit $k_{0} \rightarrow \infty$ is eventually taken.
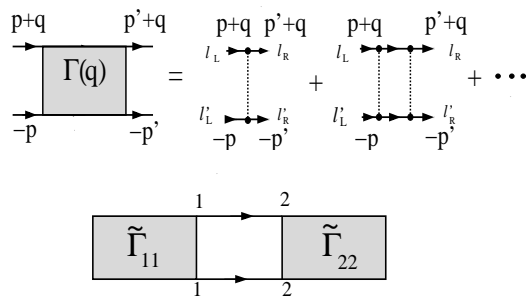

(b)

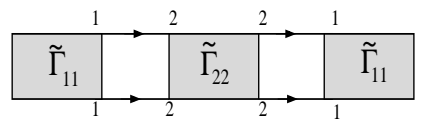

(c)

FIG. 8. (a) Particle-particle ladder in the broken-symmetry phase (conventions for four-momenta and Nambu indices are specified); (b) and (c) show typical insertions of the rungs $\Pi_{12}$ and $\Pi_{21}$, which are compatible with the regularization (A1) of the fermionic interaction potential. [Dots delimiting the potential (broken line) here represent $\tau_{3}$ Pauli matrices.]

With these premises, we consider the particle-particle ladder depicted in Fig. 8(a). ${ }^{30}$ One can convince oneself that, in these diagrams, the elementary rung

$$
K_{\ell_{L} \ell_{L}^{\prime}, \ell_{R} \ell_{R}^{\prime}}(q)=\int \frac{d \mathbf{p}}{(2 \pi)^{3}} \frac{1}{\beta} \sum_{n} \mathcal{G}_{\ell_{R} \ell_{L}}(p+q) \mathcal{G}_{\ell_{R}^{\prime} \ell_{L}^{\prime}}(-p),
$$

with the single-particle Green's functions of the BCS form (13), survives the regularization introduced by the potential (A1) in the limit $k_{0} \rightarrow \infty$ (and thus $v_{0} \rightarrow 0$ ) only if $\ell_{R}=\ell_{L}=\ell_{R}^{\prime}=\ell_{L}^{\prime}=1$ or $\ell_{R}=\ell_{L}=\ell_{R}^{\prime}=\ell_{L}^{\prime}=2$. [We use the coventions of Fig. 1 for the Nambu indices.] We then isolate the ultraviolet divergence of (A2) as $k_{0} \rightarrow \infty$, by writing [cf. Eq. (17)]

$$
\Pi_{11}(q) \equiv K_{11,11}(q)=R_{11}(q)+\frac{m k_{0}}{2 \pi^{2}}
$$

as well as

$$
\Pi_{22}(q) \equiv K_{22,22}(q)=\Pi_{11}(-q),
$$

where

$$
R_{11}(q)=\int \frac{d \mathbf{p}}{(2 \pi)^{3}}\left[\frac{1}{\beta} \sum_{n} \mathcal{G}_{11}(p+q) \mathcal{G}_{11}(-p)-\frac{m}{\mathbf{k}^{2}}\right]
$$

is a well-behaved quantity in the limit $k_{0} \rightarrow \infty$. In this way, we are led to introduce the partial ladders

$$
\begin{aligned}
\tilde{\Gamma}_{11}(q) & =-v_{0}+v_{0}^{2} \Pi_{11}(q)-v_{0}^{3} \Pi_{11}(q)^{2}+\cdots \\
& =-\frac{1}{\frac{m}{4 \pi a_{F}}+R_{11}(q)}
\end{aligned}
$$

and

$$
\begin{aligned}
\tilde{\Gamma}_{22}(q) & =-v_{0}+v_{0}^{2} \Pi_{22}(q)-v_{0}^{3} \Pi_{22}(q)^{2}+\cdots \\
& =\tilde{\Gamma}_{11}(-q) .
\end{aligned}
$$

This does not imply, however, that in Fig. 8(a) there cannot occur structures with the pair $\ell_{L}=\ell_{L}^{\prime}$ different from the pair $\ell_{R}=\ell_{R}^{\prime}$. The rungs $\Pi_{12}(q) \equiv K_{11,22}(q)$ or $\Pi_{21}(q) \equiv K_{22,11}(q)[\mathrm{cf} . \mathrm{Eq} .(18)]$ can, in fact, be inserted in between the above partial resummations $\tilde{\Gamma}(q)_{11}$ and $\tilde{\Gamma}(q)_{22}$, as indicated in Figs. 8(b) and 8(c). It is possible to take into account these structures in a systematic way, by introducing the matrix equation

$$
\begin{aligned}
& \left(\begin{array}{ll}
\Gamma_{11}(q) & \Gamma_{12}(q) \\
\Gamma_{21}(q) & \Gamma_{22}(q)
\end{array}\right)=-v_{0}\left(\begin{array}{ll}
1 & 0 \\
0 & 1
\end{array}\right) \\
& -v_{0}\left(\begin{array}{ll}
\Pi_{11}(q) & \Pi_{12}(q) \\
\Pi_{21}(q) & \Pi_{22}(q)
\end{array}\right)\left(\begin{array}{ll}
\Gamma_{11}(q) & \Gamma_{12}(q) \\
\Gamma_{21}(q) & \Gamma_{22}(q)
\end{array}\right),
\end{aligned}
$$

whose solution yields

$$
\begin{aligned}
\left(\begin{array}{ll}
\Gamma_{11}(q) & \Gamma_{12}(q) \\
\Gamma_{21}(q) & \Gamma_{22}(q)
\end{array}\right) & =\frac{1}{A(q) A(-q)-B(q)^{2}} \\
& \times\left(\begin{array}{cc}
A(-q) & B(q) \\
B(q) & A(q)
\end{array}\right)
\end{aligned}
$$

with the short-hand notation

$$
\begin{aligned}
-A(q) & =\frac{1}{v_{0}}+\Pi_{11}(q) \\
& =\int \frac{d \mathbf{p}}{(2 \pi)^{3}}\left[\frac{1}{\beta} \sum_{n} \mathcal{G}_{11}(p+q) \mathcal{G}_{11}(-p)-\frac{1}{2 E(\mathbf{p})}\right] \\
B(q) & =\Pi_{12}(q) .
\end{aligned}
$$

Note that in Eq. (A10) the gap equation

$$
-\frac{1}{v_{0}}=\int \frac{d \mathbf{p}}{(2 \pi)^{3}} \frac{1}{2 E(\mathbf{p})}
$$

has been taken into account. In this way, we can reproduce all diagrams in Fig. 8 that survive the regularization of the potential.

The expressions (A10) for $A(q)$ and $B(q)$ simplify considerably in the strong-coupling $(\beta \mu \rightarrow-\infty)$ limit we are interested in. One obtains ${ }^{37}$ :

$$
A(q) \simeq \frac{m a_{F}}{4 \pi}\left(\frac{a_{F} k_{F}^{3}}{3 \pi}-i \omega_{\nu} \frac{m}{2}+\mathbf{q}^{2} \frac{1}{8}\right)
$$

and

$$
B(q) \simeq \frac{m a_{F}^{2} k_{F}^{3}}{12 \pi^{2}}
$$

to the lowest significant order (where the Fermi wave vector $k_{F}$ is related to the density $n$ via $\left.n=k_{F}^{3} /\left(3 \pi^{2}\right)\right)$. This gives for the matrix elements (A9) the expressions: 


$$
\Gamma_{11}(q)=\Gamma_{22}(-q) \simeq \frac{8 \pi}{m^{2} a_{F}} \frac{\mu_{B}+i \omega_{\nu}+\mathbf{q}^{2} /(4 m)}{E_{B}(\mathbf{q})^{2}-\left(i \omega_{\nu}\right)^{2}}
$$

and

$$
\Gamma_{12}(q)=\Gamma_{21}(q) \simeq \frac{8 \pi}{m^{2} a_{F}} \frac{\mu_{B}}{E_{B}(\mathbf{q})^{2}-\left(i \omega_{\nu}\right)^{2}}
$$

where

$$
E_{B}(\mathbf{q})=\sqrt{\left(\frac{\mathbf{q}^{2}}{2 m_{B}}+\mu_{B}\right)^{2}-\mu_{B}^{2}}
$$

$m_{B}=2 m$ being the bosonic mass and $\mu_{B}=$ $2 k_{F}^{3} a_{F} /(3 \pi m)$ the bosonic chemical potential. Note that $\mu_{B}$ can be cast in the Bogoliubov form

$$
\mu_{B}=n_{B} v_{2}(0)=\frac{n}{2} \frac{4 \pi a_{F}}{m}
$$

in terms of the bosonic density $n_{B}=n / 2$ and of the residual bosonic interaction $v_{2}(0)=4 \pi a_{F} / m^{4,9}$ The expression (A16) has thus the form of the Bogoliubov dispersion relation ${ }^{2}$.

The results (A14) and (A15) bear strong resemblance with the normal $\left(\mathcal{G}^{\prime}\right)$ and anomalous $\left(\mathcal{G}_{21}^{\prime}\right)$ noncondensate bosonic Green's functions, respectively, within the Bogoliubov approximation, which read ${ }^{2}$ :

$$
\begin{aligned}
\mathcal{G}^{\prime}(q) & =\frac{\mu_{B}+i \omega_{\nu}+\mathbf{q}^{2} /\left(2 m_{B}\right)}{\left(i \omega_{\nu}\right)^{2}-E_{B}(\mathbf{q})^{2}} \\
\mathcal{G}_{21}^{\prime}(q) & =-\frac{\mu_{B}}{\left(i \omega_{\nu}\right)^{2}-E_{B}(\mathbf{q})^{2}} .
\end{aligned}
$$

Comparison of Eqs.(A14)-(A15) with Eqs. (A18)-(A19) thus lead us, in fact, to identify:

$$
\left\{\begin{array}{l}
\Gamma_{11}(q)=-\left(8 \pi / m^{2} a_{F}\right) \mathcal{G}^{\prime}(q) \\
\Gamma_{12}(q)=\left(8 \pi / m^{2} a_{F}\right) \mathcal{G}_{21}^{\prime}(q)
\end{array}\right.
$$

where the sign difference between the two expressions must be remarked.

Haussmann ${ }^{4}$ had already noted this sign difference and attempted to get rid of it by fixing the phase of the BCS gap function at the particular value $\pi / 2$. This prescription appears rather arbitrary, since the theory cannot depend on the value of the phase of the gap function. This sign difference is instead genuine, reflecting the fact that the particle-particle ladder propagator does not fully reduce to the Bogoliubov propagators in the strong-coupling limit.

To demonstrate this point explicitly, we resort directly to the definition of the Bogoliubov propagators ${ }^{2}$

$$
\mathcal{G}^{\prime}\left(x, x^{\prime}\right)=-\left\langle T_{\tau}\left[\Psi_{B}(x) \Psi_{B}^{\dagger}\left(x^{\prime}\right)\right]\right\rangle+\alpha(\mathbf{r}) \alpha^{*}\left(\mathbf{r}^{\prime}\right)
$$

and

$$
\mathcal{G}_{21}^{\prime}\left(x, x^{\prime}\right)=-\left\langle T_{\tau}\left[\Psi_{B}^{\dagger}(x) \Psi_{B}^{\dagger}\left(x^{\prime}\right)\right]\right\rangle+\alpha^{*}(\mathbf{r}) \alpha^{*}\left(\mathbf{r}^{\prime}\right),
$$

where $x=(\mathbf{r}, \tau)$ and $\alpha(\mathbf{r})=\left\langle\Psi_{B}(\mathbf{r})\right\rangle$ is the condensate amplitude defined in terms of the bosonic field operator $\Psi_{B}(\mathbf{r})$. The desired connection between fermionic and bosonic quantities then results by relating the bosonic operator $\Psi_{B}(\mathbf{r})$ to its fermionic counterpart $\psi_{\sigma}(\mathbf{r})$ (where $\sigma=(\uparrow, \downarrow)$ is the spin projection), by setting ${ }^{6}$ :

$$
\Psi_{B}(\mathbf{r})=\int d \rho \phi(\rho) \psi_{\downarrow}(\mathbf{r}-\rho / 2) \psi_{\uparrow}(\mathbf{r}+\rho / 2)
$$

where $\phi(\rho)$ is a suitable function. On physical grounds, we choose $\phi(\rho)$ to be the (normalized) bound-state wave function of the associated (fermionic) two-body problem:

$$
\phi(\rho)=\frac{1}{\sqrt{2 \pi a_{F}}} \frac{e^{-\rho / a_{F}}}{\rho}
$$

with Fourier transform

$$
\phi(\mathbf{p})=\sqrt{\frac{8 \pi}{a_{F}}} \frac{1}{\mathbf{p}^{2}+a_{F}^{-2}} .
$$

Entering the definition (A23) into Eqs. (A21) and (A22), and recalling the definition (5) of the two-particle correlation function, we rewrite the Bogoliubov propagators in the compact form:

$$
\mathcal{G}^{\prime}\left(\mathbf{r} \tau, \mathbf{r}^{\prime} \tau^{\prime}\right)=-\int d \rho \int d \rho^{\prime} \phi(\rho) \phi^{*}\left(\rho^{\prime}\right) L\left(1,2 ; 1^{\prime}, 2^{\prime}\right)
$$

with the identification $1=(\mathbf{r}+\rho / 2, \tau, \ell=1), 2=\left(\mathbf{r}^{\prime}-\right.$ $\left.\rho^{\prime} / 2, \tau^{\prime}, \ell=2\right), 1^{\prime}=\left(\mathbf{r}-\rho / 2, \tau^{+}, \ell=2\right)$, and $2^{\prime}=\left(\mathbf{r}^{\prime}+\right.$ $\left.\rho^{\prime} / 2, \tau^{\prime+}, \ell=1\right)$; as well as

$$
\mathcal{G}_{21}^{\prime}\left(\mathbf{r} \tau, \mathbf{r}^{\prime} \tau^{\prime}\right)=-\int d \rho \int d \rho^{\prime} \phi^{*}(\rho) \phi^{*}\left(\rho^{\prime}\right) L\left(1,2 ; 1^{\prime}, 2^{\prime}\right)
$$

with the identification $1=(\mathbf{r}-\rho / 2, \tau, \ell=2), 2=\left(\mathbf{r}^{\prime}-\right.$ $\left.\rho^{\prime} / 2, \tau^{\prime}, \ell=2\right), 1^{\prime}=\left(\mathbf{r}+\rho / 2, \tau^{+}, \ell=1\right)$, and $2^{\prime}=\left(\mathbf{r}^{\prime}+\right.$ $\left.\rho^{\prime} / 2, \tau^{\prime+}, \ell=1\right)$. In Fourier space, we write:

$$
\begin{aligned}
\mathcal{G}^{\prime}(q) & =-\int \frac{d \mathbf{p}}{(2 \pi)^{3}} \frac{1}{\beta} \sum_{n} \int \frac{d \mathbf{p}^{\prime}}{(2 \pi)^{3}} \frac{1}{\beta} \sum_{n^{\prime}} \phi(\mathbf{p}+\mathbf{q} / 2) \\
& \times \phi\left(\mathbf{p}^{\prime}+\mathbf{q} / 2\right) L_{22}^{11}\left(\mathbf{p} \omega_{n}, \mathbf{p}^{\prime} \omega_{n^{\prime}} ; q\right)
\end{aligned}
$$

and

$$
\begin{aligned}
\mathcal{G}_{21}^{\prime}(q) & =-\int \frac{d \mathbf{p}}{(2 \pi)^{3}} \frac{1}{\beta} \sum_{n} \int \frac{d \mathbf{p}^{\prime}}{(2 \pi)^{3}} \frac{1}{\beta} \sum_{n^{\prime}} \phi(\mathbf{p}+\mathbf{q} / 2) \\
& \times \phi\left(\mathbf{p}^{\prime}+\mathbf{q} / 2\right) L_{12}^{21}\left(\mathbf{p} \omega_{n}, \mathbf{p}^{\prime} \omega_{n^{\prime}} ; q\right)
\end{aligned}
$$

where the conventions for the four-momenta and Nambu indices are as in Fig. 1.

We can use at this point the expression (9) for $L$ in terms of the many-particle $T$-matrix, and limit ourselves to consider the BCS approximation (12) for the kernel $\Xi$, 
as obtained from the off-diagonal terms of the BCS selfenergy. As already stated in Section II, with this restriction only four elements of the many-particle $T$-matrix with $\ell_{L} \neq \ell_{L}^{\prime}$ and $\ell_{R} \neq \ell_{R}^{\prime}$ survive. In addition, for a contact potential the $T$-matrix in Fourier space satisfies Eq. (16), whose solution yields

$$
\begin{aligned}
\left(\begin{array}{ll}
T_{11}(q) & T_{12}(q) \\
T_{21}(q) & T_{22}(q)
\end{array}\right) & =\frac{1}{A(q) A(-q)-B(q)^{2}} \\
& \times\left(\begin{array}{cc}
-A(-q) & B(q) \\
B(q) & -A(q)
\end{array}\right)
\end{aligned}
$$

with the same definitions of Eq. (A10).

We are specifically interested in the strong-coupling limit of the above expressions, whereby the off-diagonal single-particle Green's functions can be neglected in comparison to the diagonal ones since $\Delta \ll|\mu|$ in this limit. In this way, the expressions (A28) and (A29) reduce to:

$$
\mathcal{G}^{\prime}(q) \simeq-\mathcal{F}_{2}(q)+\mathcal{F}_{1}(q)^{2} T_{11}(q)
$$

and

$$
\mathcal{G}_{21}^{\prime}(q) \simeq \mathcal{F}_{1}(q) \mathcal{F}_{1}(-q) T_{21}(q)
$$

where we have introduced the notation $(j=1,2)$

$$
\mathcal{F}_{j}(q)=\int \frac{d \mathbf{p}}{(2 \pi)^{3}} \phi(\mathbf{p}+\mathbf{q} / 2)^{j} \frac{1}{\beta} \sum_{n} \mathcal{G}_{11}(p+q) \mathcal{G}_{11}(-p) .
$$

With the approximate result

$$
\begin{aligned}
\frac{1}{\beta} \sum_{n} \mathcal{G}_{11}(p+q) \mathcal{G}_{11}(-p) & \simeq \frac{1}{E(\mathbf{k})+E(\mathbf{k}+\mathbf{q})-i \omega_{\nu}} \\
& \simeq \frac{1}{2 E(\mathbf{k})}
\end{aligned}
$$

that holds specifically in the strong coupling-limit, and using the expression (A25) for the two-body wave function, Eq. (A33) can be readily evaluated to yield:

$$
\mathcal{F}_{1}(q) \simeq \sqrt{\frac{m^{2} a_{F}}{8 \pi}}, \quad \mathcal{F}_{2}(q) \simeq \frac{m a_{F}^{2}}{4} .
$$

Neglecting the first term on the right-hand side of Eq. (A31) (which does not contain the polar structure of the $T$-matrix), we obtain eventually:

$$
\begin{aligned}
\mathcal{G}^{\prime}(q) & \simeq \frac{m^{2} a_{F}}{8 \pi} T_{11}(q) \\
\mathcal{G}_{21}^{\prime}(q) & \simeq \frac{m^{2} a_{F}}{8 \pi} T_{21}(q) .
\end{aligned}
$$

This result show that (apart from a constant prefactor) it is the $T$-matrix and not the particle-particle ladder to reduce to the Bogoliubov propagators in the strongcoupling limit of the fermionic theory. In particular, comparison of Eq. (A9) with Eq. (A30) yields Eqs. (19) of the text, thus accounting for the sign difference noted in Eqs. (A20).

Finally, it is interesting to evaluate in the strongcoupling limit also the condensate amplitude entering Eqs. (A21) and (A22), with the same approximations used above. From its definition in terms of the bosonic operator (A23), we obtain (for a homogeneous system):

$$
\begin{aligned}
\alpha & =\int d \rho \phi(\rho)\left\langle\psi_{\downarrow}(\mathbf{r}-\rho / 2) \psi_{\uparrow}(\mathbf{r}+\rho / 2)\right\rangle \\
& =\int \frac{d \mathbf{p}}{(2 \pi)^{3}} \phi(\mathbf{p}) \frac{1}{\beta} \sum_{n} e^{i \omega_{n} \eta} \mathcal{G}_{12}(p) \\
& \simeq \frac{\Delta}{2} \int \frac{d \mathbf{p}}{(2 \pi)^{3}} \frac{\phi(\mathbf{p})}{E(\mathbf{p})} \simeq \Delta \sqrt{\frac{m^{2} a_{F}}{8 \pi}} .
\end{aligned}
$$

Note that a similar relation holds (with $\alpha$ in Eq. (A37) replaced by $\sqrt{n / 2}$ ) when the diagonal BCS single-particle Green's function $\mathcal{G}_{11}$ of Eq. (13) is used to evaluate the particle density $n$ in strong coupling and at low temperature $^{37}$.

\section{APPENDIX B: MAPPING OF THE FERMIONIC ONTO THE BOSONIC DIAGRAMMATIC STRUCTURE IN THE BROKEN-SYMMETRY PHASE}

In Appendix A we have established a connection [cf. Eqs. (A20)] between the Bogoliubov propagators (A18)-(A19) and the particle-particle ladder (A14)(A15), in the strong-coupling limit. Since the Bogoliubov results (A18)-(A19) for true bosons can be obtained by considering self-energy corrections to the free-boson propagator ${ }^{35,36,2}$, it should also be possible to obtain the expressions (A14) and (A15) for the particle-particle ladder in the strong-coupling limit, by considering bosonic self-energy corrections (expressed in terms of the effective bosonic interaction in the normal phase and of the condensate density) to the particle-particle ladder in the normal phase. In this way, a mapping between the fermionic and bosonic diagrammatic structures is effectively established in the broken-symmetry phase, in a similar fashion to what has already been achieved in the normal phase ${ }^{9}$.

We begin by recalling that the Bogoliubov propagators (A18)-(A19) in the broken-symmetry phase can be obtained from the free-boson propagator $\mathcal{G}^{(0)}(q)=$ $\left(i \omega_{\nu}-\mathbf{q}^{2} /\left(2 m_{B}\right)^{2}+\mu_{B}\right)^{-1}$, by considering the following normal and anomalous bosonic self-energy corrections:

$$
\begin{aligned}
& \Sigma_{11}^{B}(q)=\Sigma_{22}^{B}(-q)=n_{0}\left(V_{B}(0)+V_{B}(\mathbf{q})\right) \\
& \Sigma_{12}^{B}(q)=\Sigma_{21}^{B}(-q)=n_{0} V_{B}(\mathbf{q})
\end{aligned}
$$

where $V_{B}(\mathbf{q})$ is the Fourier transform of the bosonic interparticle potential. Within the same approximation, the (bosonic) chemical potential is given by

$$
\mu_{B}=\Sigma_{11}^{B}(0)-\Sigma_{12}^{B}(0)=n_{0} V_{B}(0),
$$


in agreement with the Hugenholtz-Pines theorem ${ }^{2}$.

To obtain the corresponding self-energy corrections for composite bosons, it is sufficient to consider the rungs $\Pi_{11}(q)$ [cf. Eq. (17)] and $\Pi_{12}(q)$ [cf. Eq. (18)] in the strong-coupling limit. At the lowest significant order, we obtain for the contributions proportional to $\Delta^{2}$ :

$$
\begin{aligned}
& \Pi_{11}(q) \rightarrow-n_{0} m a_{F}^{2} \\
& \Pi_{12}(q) \simeq \frac{n_{0}}{2} m a_{F}^{2}
\end{aligned}
$$

where $n_{0}=|\alpha|^{2}$ is the condensate density. Recalling that in the normal phase the particle-particle ladder reduces to $^{4,9}$

$$
\Gamma^{0}(q) \simeq-\left(\frac{8 \pi}{m^{2} a_{F}}\right) \frac{1}{i \omega_{\nu}-\mathbf{q}^{2} /(4 m)^{2}+\mu_{B}}
$$

in the strong-coupling limit, the expression for $\Gamma_{11}$ containing a single insertion of $\Pi_{11}$ reads:

$$
\begin{aligned}
& \Gamma^{0}(q) \Pi_{11}(q) \Gamma^{0}(q) \\
& \quad \simeq\left(-\frac{8 \pi}{m^{2} a_{F}}\right) \frac{1}{i \omega_{\nu}-\mathbf{q}^{2} /(4 m)^{2}+\mu_{B}}\left(-n_{0} m a_{F}^{2}\right) \\
& \quad \times\left(-\frac{8 \pi}{m^{2} a_{F}}\right) \frac{1}{i \omega_{\nu}-\mathbf{q}^{2} /(4 m)^{2}+\mu_{B}} \\
& \quad=\left(-\frac{8 \pi}{m^{2} a_{F}}\right) \frac{1}{i \omega_{\nu}-\mathbf{q}^{2} /(4 m)^{2}+\mu_{B}}\left(2 n_{0} v_{2}(0)\right) \\
& \quad \times \frac{1}{i \omega_{\nu}-\mathbf{q}^{2} /(4 m)^{2}+\mu_{B}}
\end{aligned}
$$

where $v_{2}(0)=4 \pi a_{F} / m$ is the residual bosonic interaction $^{4,9}$ introduced in Eq. (A17). Apart from the overall factor $-8 \pi /\left(m^{2} a_{F}\right)$, the expression (B5) for composite bosons coincides with the lowest-order diagram obtained within the Bogoliubov approximation for true bosons, with the self-energy insertion $\Sigma_{11}(q)=2 n_{0} v_{2}(0)$ (which coincides with the first of Eqs. (B1), provided one approximates $V_{B}(\mathbf{q}) \simeq V_{B}(0) \rightarrow v_{2}(0)$ for the relevant range of wave vectors).

By a similar token, consideration of the structure of $\Gamma^{0}(q) \Pi_{12}(q) \Gamma^{0}(-q)$ leads us to identify $\Sigma_{12}(q)=n_{0} v_{2}(0)$ as the off-diagonal self-energy insertion for composite bosons to lowest order in $\Delta^{2}$. [Note that it is just the sign difference between $\Pi_{11}(q)$ and $\Pi_{12}(q)$ in Eqs. (B3) which eventually results in the sign difference between $\Gamma_{11}$ and $\Gamma_{12}$ with respect the corresponding Bogoliubov propagators, as evidenced in Eqs. (A20).]

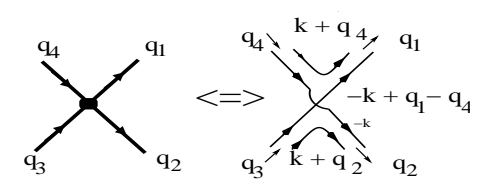

(a)

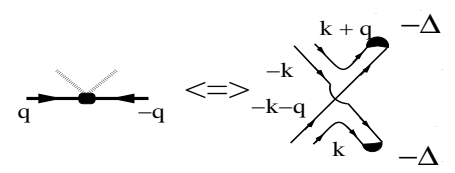

(b)

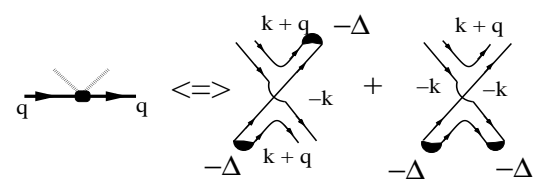

(c)

FIG. 9. Correspondence between the: (a) Effective two-boson interaction and the four-point fermionic interaction (with $\mathbf{q}_{1}+\mathbf{q}_{2}=\mathbf{q}_{3}+\mathbf{q}_{4}$ ); (b) Anomalous and (c) normal self-energies within the Bogoliubov approximation and the contractions of the four-point fermionic interaction of (a).

The above results can be interpreted euristically in terms of an effective interaction between composite bosons in the broken-symmetry phase, as follows. Let's consider the four-point vertex for composite bosons in the normal phase depicted in Fig. 9(a), which plays the role of the effective boson-boson interaction ${ }^{4,9}$ in the strongcoupling limit (we shall consider a definite choice of the spin indices).

To obtain the bosonic self-energy $\Sigma_{12}^{B}$ of Fig. 9(b) one should: $(i)$ contract the two pairs of "outgoing" connections in Fig. 9(a), by joining them through multiplication with the BCS value of $-\Delta$; (ii) multiply each fermionic single-particle line proceeding in the opposite direction (with respect to one pair of "ingoing" connections) by -1 , thus matching the standard convention [cf. the first of Eqs. (13)]. The final result corresponds to $\Pi_{12}(q)$ of Eq. (B3), since (to the lowest order in $\Delta /|\mu|) \mathcal{G}_{12}$ can be written in the form:

$$
\mathcal{G}_{12}(q) \simeq \frac{1}{i \omega_{\nu}-\xi(\mathbf{k})}(-\Delta) \frac{(-1)}{-i \omega_{\nu}-\xi(\mathbf{k})} .
$$

Note that in the above equation $\Delta$ plays the role of the square root of the condensate density (apart from a numerical coefficient), in agreement with Eq. (A37).

By a similar token, to obtain the bosonic self-energy $\Sigma_{11}^{B}$ of Fig. 9(c) one should: (i) contract one pair of "outgoing" and one pair of "ingoing" connections in Fig. 9(a), by joining them through multiplication with the BCS value of $-\Delta$ in two possible ways; (ii) multiply again each fermionic single-particle line proceeding in the opposite direction (with respect to one pair of "ingoing" connections) by -1 . In the strong-coupling limit, the final result corresponds to $-2 \Pi_{11}(q=0)$ of Eq. (B3). 


\section{APPENDIX C: EXTRACTING THE PIPPARD KERNEL FOR BOSONS WITHIN THE BOGOLIUBOV APPROXIMATION}

In this Appendix, we obtain the (wave-vector and frequency dependent) bosonic current correlation function within the Bogoliubov approximation, to which the asymptotic form of the fermionic current correlation function in the strong-coupling limit needs be compared. We shall obtain that by relying on a diagrammatic decoupling method in the broken-symmetry phase ${ }^{42}$. The static expression of this correlation function is then analyzed to extract the nonlocal part of the $\mathbf{j}$ vs $\mathbf{A}$ relation (Pippard kernel). Finally, the penetration depth obtained from Bogoliubov theory is briefly discussed.

In analogy to Eq. (10) for fermions, the following expression holds for the bosonic current correlation function:

$$
\begin{aligned}
\chi_{\gamma, \gamma^{\prime}}^{B}\left(\mathbf{r} \tau, \mathbf{r}^{\prime} \tau^{\prime}\right) & =\frac{1}{\left(2 m_{B}\right)^{2}}\left(\nabla_{\gamma}-\nabla_{\gamma}^{\prime \prime}\right)\left(\nabla_{\gamma^{\prime}}^{\prime}-\nabla_{\gamma^{\prime}}^{\prime \prime \prime}\right) \\
& \times\left\langleT _ { \tau } \left[\Psi_{B}^{\dagger}\left(\mathbf{r} \tau^{+}\right) \Psi_{B}\left(\mathbf{r}^{\prime \prime} \tau\right)\right.\right. \\
& \left.\left.\times \Psi_{B}^{\dagger}\left(\mathbf{r}^{\prime} \tau^{\prime+}\right) \Psi_{B}\left(\mathbf{r}^{\prime \prime \prime} \tau^{\prime}\right)\right]\right\rangle\left.\right|_{\mathbf{r}^{\prime \prime}=\mathbf{r}, \mathbf{r}^{\prime \prime \prime}=\mathbf{r}^{\prime}}
\end{aligned}
$$

in terms of the bosonic field operator $\Psi_{B}(\mathbf{r})$ introduced in Appendix A. Setting further

$$
\Psi_{B}(\mathbf{r})=\alpha(\mathbf{r})+\varphi(\mathbf{r})
$$

where $\alpha(\mathbf{r})=\left\langle\Psi_{B}(\mathbf{r})\right\rangle$ is the condensate amplitude and $\varphi(\mathbf{r})$ the deviation operator ${ }^{2}$, the right-hand side of Eq. (C1) is equivalent to the sum of 16 time-ordered products. With the factorization

$$
\begin{aligned}
& \left\langle T_{\tau}\left[\varphi(1) \varphi(2) \varphi^{\dagger}\left(1^{\prime}\right) \varphi^{\dagger}\left(2^{\prime}\right)\right]\right\rangle \\
& =\left\langle T_{\tau}[\varphi(1) \varphi(2)]\right\rangle\left\langle T_{\tau}\left[\varphi^{\dagger}\left(1^{\prime}\right) \varphi^{\dagger}\left(2^{\prime}\right)\right]\right\rangle \\
& +\left\langle T_{\tau}\left[\varphi(1) \varphi^{\dagger}\left(1^{\prime}\right)\right]\right\rangle\left\langle T_{\tau}\left[\varphi(2) \varphi^{\dagger}\left(2^{\prime}\right)\right]\right\rangle \\
& +\left\langle T_{\tau}\left[\varphi(1) \varphi^{\dagger}\left(2^{\prime}\right)\right]\right\rangle\left\langle T_{\tau}\left[\varphi(2) \varphi^{\dagger}\left(1^{\prime}\right)\right]\right\rangle
\end{aligned}
$$

which holds for a system of non-interacting (bosonic) quasi-particles (as described by the Bogoliubov approximation), and restricting to the case of a uniform condensate whereby $\alpha(\mathbf{r})=\alpha$, the Fourier transform Eq. (C1) becomes eventually:

$$
\begin{aligned}
& \chi_{\gamma, \gamma^{\prime}}^{B}\left(\mathbf{Q}, \Omega_{\nu}\right)=\int_{0}^{\beta} d\left(\tau-\tau^{\prime}\right) e^{i \Omega_{\nu}\left(\tau-\tau^{\prime}\right)} \int d\left(\mathbf{r}-\mathbf{r}^{\prime}\right) \\
& \quad \times e^{-i \mathbf{Q} \cdot\left(\mathbf{r}-\mathbf{r}^{\prime}\right)} \chi_{\gamma, \gamma^{\prime}}^{B}\left(\mathbf{r} \tau, \mathbf{r}^{\prime} \tau^{\prime}\right) \\
& =Q_{\gamma} Q_{\gamma^{\prime}} \frac{\alpha^{2}}{\left(2 m_{B}\right)^{2}}\left[\mathcal{G}^{\prime}(Q)+\mathcal{G}^{\prime}(-Q)-2 \mathcal{G}_{21}^{\prime}(Q)\right] \\
& -\frac{1}{\left(2 m_{B}\right)^{2}} \int \frac{d \mathbf{q}}{(2 \pi)^{3}}\left(2 q_{\gamma}+Q_{\gamma}\right)\left(2 q_{\gamma^{\prime}}+Q_{\gamma^{\prime}}\right) \frac{1}{\beta} \sum_{\omega_{\nu}} \\
& \times\left[\mathcal{G}^{\prime}(q) \mathcal{G}^{\prime}(q+Q)-\mathcal{G}_{21}^{\prime}(q) \mathcal{G}_{21}^{\prime}(q+Q)\right] .
\end{aligned}
$$

Utilizing at this point the form (A18) and (A19) of the Bogoliubov propagators, the result (C4) is seen to coincide with the one obtained alternatively by exploiting the Lehmann representation of the current correlation function $^{42}$. Although corrections to the Bogoliubov approximation for the current correlation function (as to make the longitudinal f-sum rule satisfied while preserving the form of the Bogoliubov propagators) have been considered in a formal way ${ }^{43}$, in this paper we restrict ourselves realistically to the simplest approximation (C4) for the bosonic limit, since we are ultimately interested in the evolution of the fermionic current correlation function from weak to strong coupling.

The "static" $\left(\mathbf{Q} \rightarrow 0, \Omega_{\nu}=0\right)$ limit of Eq. (C4) can be used to test explicitly the longitudinal f-sum rule (15) and to obtain the (temperature dependence of the) superfluid density via Eq.(14). One obtains:

$$
\begin{aligned}
\chi_{\gamma, \gamma^{\prime}}^{B}(\mathbf{Q} \rightarrow & \left.0, \Omega_{\nu}=0\right) \\
& =-\frac{n_{0}}{2 m_{B}^{2}} \lim _{\mathbf{Q} \rightarrow 0} Q_{\gamma} Q_{\gamma^{\prime}} \frac{\left(u_{B}(\mathbf{Q})+v_{B}(\mathbf{Q})\right)^{2}}{E_{B}(\mathbf{Q})} \\
& +\int \frac{d \mathbf{q}}{(2 \pi)^{3}} \frac{q_{\gamma}}{m_{B}} \frac{q_{\gamma^{\prime}}}{m_{B}} \frac{\partial n_{B}\left(E_{B}(\mathbf{q})\right)}{\partial E_{B}(\mathbf{q})}
\end{aligned}
$$

where $n_{B}(E)=\left(e^{\beta E}-1\right)^{-1}$ is the Bose-Einstein distribution function, $2 v_{B}(\mathbf{q})^{2}=\left[\left(\mathbf{q}^{2} /\left(2 m_{B}\right)+\right.\right.$ $\left.\left.n_{0} V_{B}(\mathbf{q})\right) / E_{B}(\mathbf{q})-1\right]$ and $u_{B}(\mathbf{q})^{2}-v_{B}(\mathbf{q})^{2}=1, V_{B}(\mathbf{q})$ here being the same bosonic potential of Appendix B. Recalling that $v_{B}(\mathbf{q})^{2} \simeq m s /(2|\mathbf{q}|)$ and $E_{B}(\mathbf{q}) \simeq s|\mathbf{q}|$ in the small-q limit (where $s=\sqrt{n_{0} V_{B}(0) / m_{B}}$ is the sound velocity), the first term on the right-hand side of Eq. (C5) reduces to $-\hat{Q}_{\gamma} \hat{Q}_{\gamma^{\prime}} n_{0} / m_{B}$, implying that the f-sum rule (15) is only approximately verified, insofar as $n_{0} \simeq n_{B}$ within the Bogoliubov approximation. The second term on the right-hand side of Eq. (C5), on the other hand, has the form of $\left(-1 / m_{B}\right.$ times $)$ the expression of the Landau's normal-fluid density $\rho_{n}=n-\rho_{s}$ (cf., e.g., Ref. 2), and is also temperature dependent (albeit through the Bogoliubov expression of the quasi-particle excitation energy).

In addition, the Pippard kernel can be obtained from Eq. (C4) by letting $\Omega_{\nu}=0$ but keeping $\mathbf{Q}$ finite, in analogy to the BCS treatment for fermions ${ }^{1,2}$. In particular, for a transverse vector potential in the zero-temperature limit one considers the expression:

$$
\begin{aligned}
& \int \frac{d \mathbf{Q}}{(2 \pi)^{3}} e^{i \mathbf{R} \cdot \mathbf{Q}} \chi_{\gamma, \gamma^{\prime}}^{B}\left(\mathbf{Q}, \Omega_{\nu}=0\right) \\
& \quad=\frac{1}{2 m_{B}^{2}(2 \pi)^{4}} \int_{0}^{\infty} d q q \int_{0}^{\infty} d p p G^{B}(q, p) \\
& \quad \times \quad \lim _{\mathbf{R}^{\prime} \rightarrow 0} \frac{\partial}{\partial R_{\gamma}^{\prime}} \frac{\partial}{\partial R_{\gamma^{\prime}}^{\prime}} j_{0}\left(q\left|\mathbf{R}^{\prime}-\mathbf{R}\right|\right) j_{0}\left(p\left|\mathbf{R}^{\prime}+\mathbf{R}\right|\right)
\end{aligned}
$$

with the notation $q=|\mathbf{q}|, p=|\mathbf{p}|$, and

$$
\begin{aligned}
& G^{B}(q, p)=\frac{q p}{E_{B}(q) E_{B}(p)\left(E_{B}(q)+E_{B}(p)\right)}\left[\epsilon_{B}(q) \epsilon_{B}(p)\right. \\
& \left.+n_{0} V_{B}(q) \epsilon_{B}(p)+n_{0} V_{B}(p) \epsilon_{B}(q)-E_{B}(q) E_{B}(p)\right]
\end{aligned}
$$

where $\epsilon_{B}(q)=q^{2} /\left(2 m_{B}\right)$ and $j_{0}(z)=(\sin z) / z$ is the spherical Bessel function of zeroth order. The function 
$G^{B}(q, p)$ (plotted in Fig. 10) is symmetric under the interchange of $q$ and $p$, vanishes for $q=p$ but is elsewhere positive, and its relevant range extends about $q \sim \xi_{\text {phase }}^{-1}$ and $p \sim \xi_{\text {phase }}^{-1}$, where

$$
\xi_{\text {phase }}=\frac{1}{\sqrt{4 m_{B} n_{0} V_{B}(0)}}
$$

is the characteristic length of the Bose gas associated with the chemical potential $\mu_{B}=n_{0} V_{B}(0)$ [cf. Eq. (A17)] which represents the only energy scale in the problem ${ }^{44}$.

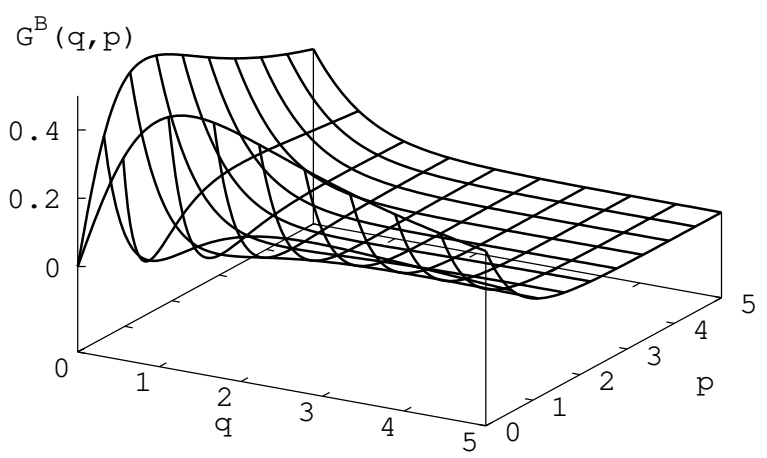

FIG. 10. Two-dimensional plot of the function $G^{B}(q, p)$ defined by Eq. (C7), with the wave vectors in units of $\left(\sqrt{2} \xi_{\text {phase }}\right)^{-1}$.

For the purpose of extracting the leading behavior of the function (C6) for large values of $R=|\mathbf{R}|$, the spatial derivatives therein can be calculated with the same approximations exploited in the analogous calculation for the BCS (bubble) contribution to the current correlation function $^{1,2}$. One obtains:

$$
\begin{aligned}
\lim _{\mathbf{R}^{\prime} \rightarrow 0} & \frac{\partial}{\partial R_{\gamma}^{\prime}} \frac{\partial}{\partial R_{\gamma^{\prime}}^{\prime}} j_{0}\left(q\left|\mathbf{R}^{\prime}-\mathbf{R}\right|\right) j_{0}\left(p\left|\mathbf{R}^{\prime}+\mathbf{R}\right|\right) \\
\approx & -2 \frac{R_{\gamma} R_{\gamma^{\prime}}}{R^{4}} \cos (q-p) R
\end{aligned}
$$

Introducing the notation

$$
g^{B}(R)=\int_{0}^{\infty} d q q \int_{0}^{\infty} d p p G^{B}(q, p) \cos (q-p) R,
$$

Eq. (C6) acquires eventually the form:

$$
\begin{array}{r}
\int \frac{d \mathbf{Q}}{(2 \pi)^{3}} e^{i \mathbf{R} \cdot \mathbf{Q}} \chi_{\gamma, \gamma^{\prime}}^{B}\left(\mathbf{Q}, \Omega_{\nu}=0\right) \\
\approx-\frac{1}{(2 \pi)^{4} m_{B}^{2}} \frac{R_{\gamma} R_{\gamma^{\prime}}}{R^{4}} g^{B}(R) .
\end{array}
$$

It should be noted that

$$
\int_{0}^{\infty} d R g^{B}(R)=\pi \int_{0}^{\infty} d q q^{2} G^{B}(q, q)=0
$$

since $G^{B}(q, q)=0$ for all values of $q$.

In practice, it is convenient to extract at the ouset a delta-function contribution from the function $g^{B}(R)$, by setting

$$
g^{B}(R)=g_{n}^{B}(R)-2 \mathcal{I}^{B} \delta(R)
$$

where

$$
\mathcal{I}^{B}=\int_{0}^{\infty} d R g_{n}^{B}(R)
$$

and evaluate the remainder $g_{n}^{B}(R)$ numerically. To this end, we exploit the asymptotic form of the function $G^{B}(q, p)$ and set

$$
f^{B}(\tilde{q}) \equiv \lim _{\tilde{p} \rightarrow \infty} \tilde{p} G^{B}(\tilde{q}, \tilde{p})=2 m_{B} \frac{\left(\tilde{q}^{2}+1-\sqrt{\tilde{q}^{4}+2 \tilde{q}^{2}}\right)}{\sqrt{\tilde{q}^{2}+2}}
$$

where $\tilde{q}=q \sqrt{2} \xi_{\text {phase }}$ and $\tilde{p}=p \sqrt{2} \xi_{\text {phase. }}$. With the further notation

$$
\bar{G}^{B}(\tilde{q}, \tilde{p}) \equiv \tilde{q} G^{B}(\tilde{q}, \tilde{p}) \tilde{p}-\tilde{q} f^{B}(\tilde{q})-\tilde{p} f^{B}(\tilde{p}),
$$

we obtain:

$$
\begin{aligned}
g_{n}^{B}(R) & =\frac{1}{4 \xi_{\text {phase }}^{4}}\left\{\int_{0}^{\infty} d \tilde{q} \int_{0}^{\infty} d \tilde{p} \bar{G}^{B}(\tilde{q}, \tilde{p}) \cos (\tilde{q}-\tilde{p}) \tilde{R}\right. \\
& \left.+\frac{2}{\tilde{R}} \int_{0}^{\infty} d \tilde{q} \tilde{q} f^{B}(\tilde{q}) \sin (\tilde{q} \tilde{R})\right\}
\end{aligned}
$$

where $\tilde{R}=R /\left(\sqrt{2} \xi_{\text {phase }}\right)$, as well as

$$
\mathcal{I}^{B}=-\frac{\pi \sqrt{2}}{4 \xi_{\text {phase }}^{3}} \int_{0}^{\infty} d \tilde{q} \tilde{q} f^{B}(\tilde{q})=-\frac{\pi m_{B}}{3 \xi_{\text {phase }}^{3}} .
$$

The function $g_{n}^{B}(R)$ (plotted in Fig. 11) is negative, diverges like $m_{B}(\pi-1) /\left(2 \xi_{\text {phase }}\right) \ln \tilde{R}$ for $\tilde{R} \rightarrow 0$, is strongly localized over the range $\xi_{\text {phase }}$, and shows minor oscillations about zero for $R>\xi_{\text {phase }}$.

In contrast to an analogous procedure within the fermionic BCS approximation of Appendix D, the strength $\mathcal{I}^{B}$ of the delta function in Eq. (C13) depends on the condensate density via $\xi_{\text {phase }}$ (in particular, the strength of the delta function and consequently the area enclosed by $g_{n}^{B}$ vanish in the limit $\xi_{\text {phase }} \rightarrow \infty$, corresponding to noninteracting (condensed) bosons). Accordingly, the delta-function contribution does not cancel the diamagnetic term in the relation between the induced current $\mathbf{j}$ and the vector potential $\mathbf{A}$, as it happens instead for the BCS case ${ }^{2}$ (cf. also Appendix D). In the present case, in fact, one obtains: 


$$
\begin{aligned}
j_{\gamma}(\mathbf{r}) & =-\frac{1}{m_{B} c}\left(n_{B}-\frac{1}{36 \pi^{2} \xi_{\text {phase }}^{3}}\right) A_{\gamma}(\mathbf{r}) \\
& +\frac{1}{(2 \pi)^{4} m_{B}^{2} c} \int d \mathbf{r}^{\prime} \frac{R_{\gamma} \sum_{\gamma^{\prime}} R_{\gamma^{\prime}} A_{\gamma^{\prime}}\left(\mathbf{r}^{\prime}\right)}{R^{4}} g_{n}^{B}(R)
\end{aligned}
$$

where $\mathbf{R}=\mathbf{r}-\mathbf{r}^{\prime}$ and $c$ is the light velocity. Note how the form (C19) for the current response kernel is intermediate between the (local) London's form and the (nonlocal) Pippard's form ${ }^{2}$. Note also that, in the noninteractingboson limit (whereby $\xi_{\text {phase }} \rightarrow \infty$ ), Eq.(C19) correctly reduces to the local London's form

$$
j_{\gamma}(\mathbf{r})=-\frac{n_{B}}{m_{B} c} A_{\gamma}(\mathbf{r})
$$

in agreement with the result obtained in Ref. 45 for a condensed Bose gas at zero temperature (for fermions within the BCS approximation, on the other hand, only the nonlocal Pippard's term survives ${ }^{2}$ ).

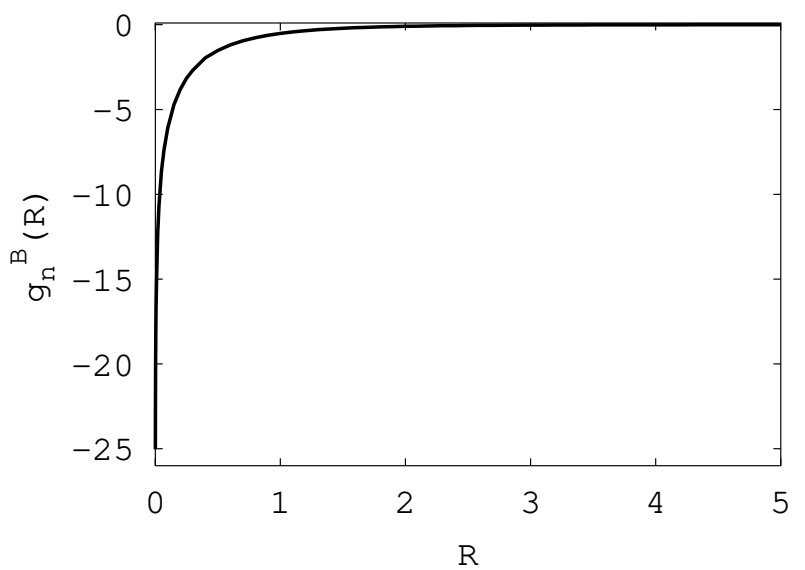

FIG. 11. Plot of the function $g_{n}^{B}(R)$ (in units of $\left.m_{B} /\left(4 \xi_{\text {phase }}^{4}\right)\right)$ as determined numerically from Eq. (C17), vs $R$ (in units of $\sqrt{2} \xi_{\text {phase }}$ ).

Finally, the real-space relation (C19) can be transposed to a local condition in wave-vector space as follows. Taking $\mathbf{q} / / \hat{z}$ and $\mathbf{A}(\mathbf{q}) / / \hat{y}$ ( $\operatorname{such}$ that $\mathbf{q} \cdot \mathbf{A}(\mathbf{q})=0$ in the London gauge), we obtain:

$$
j_{y}(\mathbf{q})=-\frac{c}{4 \pi} K(q) A_{y}(\mathbf{q})
$$

where

$$
-\frac{c}{4 \pi} K(q)=-\frac{1}{m_{B} c}\left(n_{B}-\frac{1}{36 \pi^{2} \xi_{\text {phase }}^{3}}\right)+Q_{y y}(q)
$$

and

$$
Q_{y y}(q)=-\frac{1}{4 \pi^{3} m_{B}^{2} c} \int_{0}^{\infty} d r \frac{g_{n}^{B}(r)}{r^{2}} \frac{1}{q} \frac{\partial}{\partial q}\left(\frac{\sin q r}{q r}\right) .
$$

Following Pippard's treatment ${ }^{2,26}$, we can then define the penetration depth via the relation:

$$
\lambda=\frac{2}{\pi} \int_{0}^{\infty} d q \frac{1}{q^{2}+K(q)} .
$$

This expression has to be compared with London's relation at zero temperature

$$
\lambda_{L}=\sqrt{\frac{m_{B} c^{2}}{4 \pi n_{B} e_{B}^{2}}}=\sqrt{\frac{m c^{2}}{4 \pi n e^{2}}}
$$

(where the electric charge $e_{B}=2 e$ has been restored for convenience). To this end, we follow an analogous treatment within BCS theory ${ }^{2}$ and consider the local $\left(\lambda \gg \xi_{\text {phase }}\right)$ and nonlocal $\left(\lambda \ll \xi_{\text {phase }}\right)$ limits separately, by analyzing the $q$-dependence of the kernel $(\mathrm{C} 22)$. Note that in the present case, while the ratio $Q_{y y}(q) / Q_{y y}(0)$ is a universal function of $\tilde{q}=q \sqrt{2} \xi_{\text {phase }}$, the ratio $K(q) / K(0)$ is not, since its asymptotic limit $K(\infty) / K(0)=1-\left(36 \pi^{2} n_{B} \xi_{\text {phase }}^{3}\right)^{-1}\left(\right.$ with $\left.K(0)=\lambda_{L}^{-2}\right)$ is nonvanishing. This contrasts the situation in BCS theory where $K(\infty)=0$, the difference originating from the fact that in Bogoliubov theory cancellation of the diamagnetic term in Eq. (C22) does not occur. In the local $\left(\lambda \gg \xi_{\text {phase }}\right)$ limit, whereby we may approximate $K(q)$ by $K(0)$ in Eq. (C24), we obtain $\lambda \simeq \lambda_{L}$. In the nonlocal $\left(\lambda \ll \xi_{\text {phase }}\right)$ limit, on the other hand, whereby we may approximate $K(q)$ by $K(\infty)$ in Eq. (C24), we obtain $\lambda \simeq \lambda_{L}(K(\infty) / K(0))^{-1 / 2} \simeq \lambda_{L}$ owing to the diluteness condition $^{46}$. Within the Bogoliubov theory, there is thus no way to get from Eq. (C24) values for $\lambda$ significantly different from London's value $\lambda_{L}$, in contrast with BCS theory $^{2}$ whereby one obtains $\lambda \gg \lambda_{L}$ in the nonlocal limit.

\section{APPENDIX D: EXTRACTING THE PIPPARD KERNEL FOR SUPERCONDUCTING FERMIONS WITHIN THE BCS APPROXMATION}

In this Appendix, we set up a general procedure to extract the Pippard kernel from the BCS bubble contribution to the current correlation function. Contrary to the method introduced by BCS theory to deal with the (extreme) weak-coupling limit ${ }^{2}$, our procedure is applicable to all couplings. Although some expressions reported in this Appendix are similar to those of Appendix C for the bosonic counterpart, we shall report them here to point out similarities and differences between the two cases.

In the BCS method ${ }^{2}$, a local (delta-function) contribution is identified from the paramagnetic contribution to the current induced by a (transverse) vector potential. This local contribution is shown to cancel completely the diamagnetic term, thus making "nonlocal" the relation between the current and the vector potential in coordinate space (over the scale of the Pippard coherence length 
$\left.\xi_{0}\right)$. We will show in the following that this cancellation of the diamagnetic term by the local term originating from the paramagnetic contribution is, strictly speaking, never complete. Yet, it can be considered approximately complete insofar as the length scale $k_{F}^{-1}$ is much smaller than $\xi_{0}$ (a situation which certainly applies to the extreme weak-coupling limit). At stronger coupling (when $k_{F}^{-1}$ becomes comparable with $\xi_{0}$ ), on the other hand, this argument is bound to fail. As a consequence, the relation between the current and the vector potential in coordinate space contains a nonlocal as well as a local contribution.

Quite generally, the current correlation function at zero frequency obtained from the BCS bubble can be cast in the form:

$$
\begin{aligned}
\chi_{\gamma, \gamma^{\prime}}^{B C S}\left(\mathbf{Q}, \Omega_{\nu}=0\right) & =-\frac{1}{(2 m)^{2}} \int \frac{d \mathbf{q}}{(2 \pi)^{3}}\left(2 q_{\gamma}+Q_{\gamma}\right) \\
& \times\left(2 q_{\gamma^{\prime}}+Q_{\gamma^{\prime}}\right) \frac{G^{B C S}(\mathbf{q}, \mathbf{q}+\mathbf{Q})}{2|\mathbf{q}||\mathbf{q}+\mathbf{Q}|}
\end{aligned}
$$

where (cf., e.g., Ref. 2)

$$
G^{B C S}(q, p)=-2 q p \frac{\xi(q) \xi(p)+\Delta^{2}-E(q) E(p)}{E(q) E(p)(E(q)+E(p))} .
$$

Note that, similarly to the function $G^{B}(q, p)$ of Eq. (C7), the function $G^{B C S}(q, p)$ is also symmetric under the interchange of $p$ and $q$, vanishes for $p=q$, and is elsewhere positive.

In coordinate space we write correspondingly [cf. Eq. (C6)]:

$$
\begin{aligned}
& \int \frac{d \mathbf{Q}}{(2 \pi)^{3}} e^{i \mathbf{R} \cdot \mathbf{Q}} \chi_{\gamma, \gamma^{\prime}}^{B C S}\left(\mathbf{Q}, \Omega_{\nu}=0\right) \\
& \quad=\frac{1}{2 m^{2}(2 \pi)^{4}} \int_{0}^{\infty} d q q \int_{0}^{\infty} d p p G^{B C S}(q, p) \\
& \quad \times \quad \lim _{\mathbf{R}^{\prime} \rightarrow 0} \frac{\partial}{\partial R_{\gamma}^{\prime}} \frac{\partial}{\partial R_{\gamma^{\prime}}^{\prime}} j_{0}\left(q\left|\mathbf{R}^{\prime}-\mathbf{R}\right|\right) j_{0}\left(p\left|\mathbf{R}^{\prime}+\mathbf{R}\right|\right) .
\end{aligned}
$$

As in Appendix C, for the purpose of extracting the leading behavior of the function (D3) for large values of $R=|\mathbf{R}|$, we can make the approximation (C9) in Eq. (D3).

Introducing further the notation [cf. Eq. (C10)]:

$$
g^{B C S}(R)=\int_{0}^{\infty} d q q \int_{0}^{\infty} d p p G^{B C S}(q, p) \cos (q-p) R,
$$

Eq. (D3) acquires eventually the form [cf. Eq. (C11)]:

$$
\begin{gathered}
\int \frac{d \mathbf{Q}}{(2 \pi)^{3}} e^{i \mathbf{R} \cdot \mathbf{Q}} \chi_{\gamma, \gamma^{\prime}}^{B C S}\left(\mathbf{Q}, \Omega_{\nu}=0\right) \\
\approx-\frac{1}{(2 \pi)^{4} m^{2}} \frac{R_{\gamma} R_{\gamma^{\prime}}}{R^{4}} g^{B C S}(R) .
\end{gathered}
$$

It should be noted that also in this case

$$
\int_{0}^{\infty} d R g^{B C S}(R)=\pi \int_{0}^{\infty} d q q^{2} G^{B C S}(q, q)=0
$$

since $G^{B C S}(q, q)=0$ for all values of $q$.

The integral in Eq. (D4) must be treated with care, since the product $p G^{B C S}(q, p)$ does not vanish in the limit of large $p$, thus implying a delta-function contribution to the function $g^{B C S}(R)$ as for the boson case of Appendix C. To extract this contribution, we exploit the asymptotic form of the function $G^{B C S}(q, p)$ and set [cf. Eq. (C15)]

$$
f^{B C S}(q) \equiv \lim _{p \rightarrow \infty} p G^{B C S}(q, p)=8 m q v_{q}^{2}
$$

where $v_{q}^{2}=(1-\xi(q) / E(q)) / 2$ is the usual BCS factor. With the further notation

$$
\bar{G}^{B C S}(q, p) \equiv q G^{B C S}(q, p) p-q f^{B C S}(q)-p f^{B C S}(p),
$$

Eq. (D4) becomes [cf. Eq. (C13)]:

$$
g^{B C S}(R)=g_{n}^{B C S}(R)-2 \mathcal{I}^{B C S} \delta(R)
$$

where [cf. Eq. (C17)]

$$
\begin{aligned}
g_{n}^{B C S}(R) & =\int_{0}^{\infty} d q \int_{0}^{\infty} d p \bar{G}^{B C S}(q, p) \cos (q-p) R \\
& +\frac{2}{R} \int_{0}^{\infty} d q q f(q) \sin (q R)
\end{aligned}
$$

and

$$
\mathcal{I}^{B C S}=-\pi \int_{0}^{\infty} d q q f^{B C S}(q)=-8 \pi^{3} m n,
$$

$n$ being the particle density. Note that $\mathcal{I}^{B C S}$ is a constant, while its bosonic counterpart of Eq. (C18) depend on the bosonic interaction. In terms of the above quantities, the relation between the induced current $\mathbf{j}$ and the vector potential A becomes eventually:

$$
\begin{aligned}
j_{\gamma}(\mathbf{r}) & =-\frac{1}{m c}\left(n+\frac{\mathcal{I}^{B C S}}{12 \pi^{3} m}\right) A_{\gamma}(\mathbf{r}) \\
& +\frac{1}{(2 \pi)^{4} m^{2} c} \int d \mathbf{r}^{\prime} \frac{R_{\gamma} \sum_{\gamma^{\prime}} R_{\gamma^{\prime}} A_{\gamma^{\prime}}\left(\mathbf{r}^{\prime}\right)}{R^{4}} g_{n}^{B C S}(R)
\end{aligned}
$$

in the place of Eq. (C19) for bosons. Insertion of the value (D11) into the first term on the right-hand side of Eq. (D12) does not lead to a complete cancellation of the diamagnetic term, since $n+\mathcal{I}^{B C S} /\left(12 \pi^{3} m\right)=n / 3$ in this case. In the standard BCS treatment ${ }^{2}$, on the other hand, it is claimed that the diamagnetic term is completely cancelled by the local (delta-function) contribution originating from the paramagnetic term. This apparent contradiction is resolved by the following argument. 
A crucial point in our analysis is that $g_{n}^{B C S}(R)$ is a regular function of $R$, in the sense that it does not contribute any additional delta-function term to the function $g^{B C S}(R)$, besides the one explicitly indicated in Eq. (D9). By a careful analysis of the one- and two-dimensional integrals entering the definition (D10), it can, in fact, be shown that $g_{n}^{B C S}(R)$ contains at most the following (integrable) logarithmic singularity

$$
g_{n}^{B C S}(R) \approx-16(\pi-1) m^{3} \Delta^{2} \ln \left(\frac{1}{R k_{F}}\right)
$$

for $R k_{F} \ll 1$. Note that the form of this singularity is universal, in the sense that it depends on the coupling value only through the value of the gap function $\Delta$ in the prefactor.

To the singular term (D13) one should add nonsingular contributions that depend on the value of the coupling parameter. In particular, for $R k_{F} \ll 1$ in weak coupling one obtains the nonsingular contribution $4 m k_{F}^{4}$. This implies that, in weak coupling, the appearance of the singular term (D13) is restricted to extremely small values of $R$, and it cannot be detected for all practical purposes. For instance, when $k_{F} \xi_{0}=k_{F}^{2} /(\pi m \Delta)=32$ (so that the extreme weak-coupling limit is definitively reached), one obtains that the singular term (D13) dominates over the constant contribution $4 m k_{F}^{4}$ only for $k_{F} R \lesssim \exp (-1000)$. At larger couplings, however, the singular term (D13) becomes non negligible for appreciable values of $k_{F} R$, as seen in Fig. 7 of the text. [We prefer in this Appendix to use the Pippard coherence length $\xi_{0}$ instead of $\xi_{\text {pair }}$ to make easier the comparison with the BCS method to obtain the Pippard kernel in the extreme weak-coupling limit.]

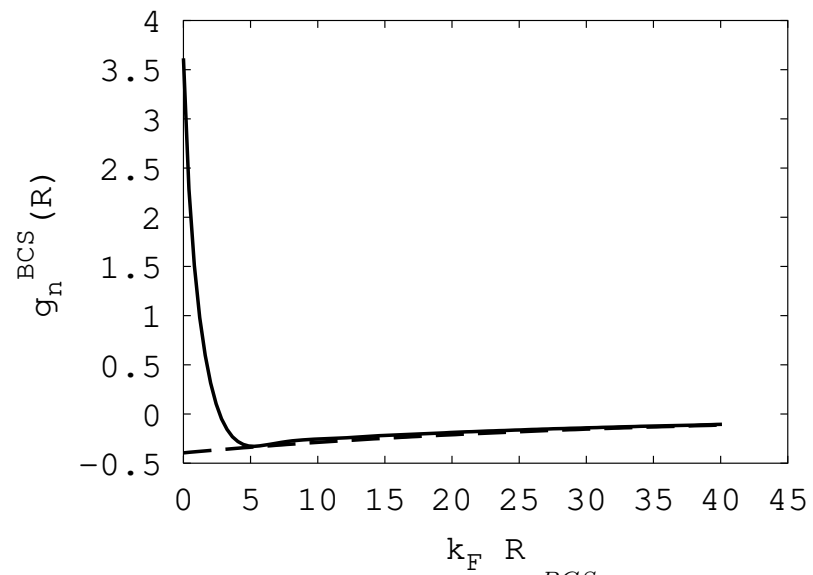

FIG. 12. Plot of the function $g_{n}^{B C S}(R)$ (in units of $\left.m k_{F}^{4}\right)$ as determined numerically from Eq. (D4), vs $k_{F} R$ for $k_{F} \xi_{0}=32$ (full line). The corresponding function $-\left(2 m \pi k_{F}\right)^{2} \Delta \exp \left(-R / \xi_{0}\right)$ is also shown for comparison (dashed line).

A plot of the function $g_{n}^{B C S}(R)$ (in units of $m k_{F}^{4}$ ) vs $k_{F} R$ obtained by numerical evaluation of Eq. (D10) is shown in Fig. 12 (full line) for $k_{F} \xi_{0}=32$, whereby the singular behavior (D13) is not visible by the above argument. From this plot, the two characteristic length scales of BCS theory (namely, $k_{F}^{-1}$ and $\xi_{0}$ such that $k_{F}^{-1} \ll \xi_{0}$ in the weak-coupling limit) are evident. Specifically, from the plot of $g_{n}^{B C S}(R)$ one can isolate its "tail" represented by the function $-\left(2 m \pi k_{F}\right)^{2} \Delta \exp \left(-R / \xi_{0}\right)$ (or, better, by the function $J(R)$ of BCS theory obtained by integrating the Bessel function of imaginary argument ${ }^{2}$ ), shown in Fig. 12 by the dashed line. Once this tail has been isolated, the remainder $g_{n}^{r e m}(R)=$ $g_{n}(R)+\left(2 m \pi k_{F}\right)^{2} \Delta \exp \left(-R / \xi_{0}\right)$ is localized on the scale $k_{F}^{-1}$, which is much smaller than the scale $\xi_{0}$ of the tail in the weak-coupling limit. For these reasons, the remainder itself can be effectively assimilated to a deltafunction contribution with finite strength (by setting $g_{n}^{r e m}(R)=-2 \mathcal{I}^{\prime} \delta(R)$ ), which thus adds up to the true delta-function contribution of Eq. (D9). Note that, to cancel completely the diamagnetic term in Eq. (D12), the factor $\mathcal{I}^{\prime}$ should equal $-4 \pi^{3} m n$. This value is indeed obtained by the data of Fig. 12 within a $10^{-3}$ relative error.

Our procedure has to be contrasted with the standard BCS treatment in the weak-coupling limit ${ }^{2}$, whereby the delta-function contribution that cancels completely the diamagnetic term in the $\mathbf{j}$ vs $\mathbf{A}$ relation is obtained by letting the lower limit of an integration over $\xi(p)$ and $\xi(q)$ going to $-\infty$ (instead of holding it at the original finite value $\left.-k_{F}^{2} /(2 m)\right)$.

Although the two procedures yield correctly the same results in the weak-coupling limit, our procedure can be readily extended to the intermediate-coupling region where the two length scales $k_{F}^{-1}$ and $\xi_{0}$ becomes comparable, as shown in Section IV.

${ }^{1}$ J.R. Schrieffer, Theory of Superconductivity (W.A. Benjamin, New York, 1964), Chapter 8.

2 A.L. Fetter and J.D. Walecka, Quantum Theory of ManyParticle Systems (McGraw-Hill, New York, 1971).

${ }^{3}$ M. Randeria, J.-M. Duan, L.-Y. Shieh, Phys. Rev. B 41, 327 (1990).

${ }^{4}$ R. Haussmann, Z. Phys. B 91, 291 (1993).

${ }^{5}$ F. Pistolesi and G.C. Strinati, Phys. Rev. B 49, 6356 (1994).

${ }^{6}$ F. Pistolesi and G.C. Strinati, Phys. Rev. B 53, 15168 (1996).

${ }^{7}$ B. Jankó, J. Maly, and K. Levin, Phys. Rev. B 56, R11407 (1997).

${ }^{8}$ S. Stintzing and W. Zwerger, Phys. Rev. B 56, 9004 (1997).

${ }^{9}$ P. Pieri and G.C. Strinati, Phys. Rev. B 61, 15370 (2000), and cond-mat/9811166.

${ }^{10}$ H. Ding et al., Nature 382, 51 (1996); and Phys. Rev. Lett. 78, 2628 (1997). 
${ }^{11}$ A. G. Loeser et al., Science 273, 325 (1996).

${ }^{12}$ Y. J. Uemura et al., Phys. Rev. Lett. 62, 2317 (1989).

${ }^{13}$ D.M. Eagles, Phys. Rev. 186, 456 (1969).

14 A.J. Leggett, in Modern Trends in the Theory of Condensed Matter, edited by A. Pekalski and R. Przystawa, Lecture Notes in Physics Vol.115 (Springer-Verlag, Berlin, 1980), p.13.

${ }^{15}$ P. Nozières and S. Schmitt-Rink, J. Low. Temp. Phys. 59, 195 (1985).

${ }^{16}$ R. Haussmann, Phys. Rev. B 49, 12975 (1994).

17 A. Perali, P. Pieri, G.C. Strinati, and C. Castellani, Phys. Rev. B 66, 024510 (2002).

${ }^{18}$ P. Pieri, L. Pisani, and G.C. Strinati (unpublished).

${ }^{19}$ P. Pieri, G.C. Strinati, and D. Moroni, Phys. Rev. Lett. 89, 127003 (2002).

${ }^{20}$ G.C. Strinati, P. Pieri, and C. Lucheroni, Eur. Phys. J. B 30, 161 (2002).

${ }^{21}$ M. Luban and W.D. Grobman, Phys. Rev. Lett. 17, 182 (1966); see also M. Luban in Quantum Fluids, N. Wiser and D.J. Amit, Eds. (Gordon and Breach, New York, 1970), p.117.

${ }^{22}$ G.C. Strinati, in Electrons and Photons in Solids, a Volume in honour of Franco Bassani (Scuola Normale Superiore, Pisa, 2001), p.403.

23 P.C. Hohenberg and P.C Martin, Ann. Phys. 34, 291 (1965).

24 A. Brunello, F. Dalfovo, L. Pitaevskii, and S. Stringari, Phys. Rev. Lett. 85, 4422 (2000); J. M. Vogels, K. Xu, C. Raman, J. R. Abo-Shaeer, and W. Ketterle, Phys. Rev. Lett. 88, 060402 (2002).

${ }^{25}$ L.G. Aslamazov and A.I. Larkin, Sov. Phys. JETP 10, 875 (1968).

26 A.B. Pippard, Proc. Roy. Soc. (London) A216, 547 (1953); T.E. Faber and A.B. Pippard, Proc. Roy. Soc. (London) A231, 336 (1955).

${ }^{27}$ L. Benfatto, A. Toschi, S. Caprara, and C. Castellani, Phys. Rev. B 66, 054515 (2002).

${ }^{28}$ G. Baym, Phy. Rev. 127, 1391 (1962).

${ }^{29}$ G. Strinati, La Rivista del Nuovo Cimento Vol.11, N.12, (1988).

30 The arrows attached to the Nambu Green's functions have the meaning of pointing from the second to the first argument of the Green's functions. The distinction between particle-particle and particle-hole diagrams is, however, purely conventional since particle and hole modes get intimately interrelated in the broken-symmetry phase. We shall nevertheless mantain the terminology used for the normal phase and refer, for instance, to the diagrams of Fig. 8(a) as to the "particle-particle" ladder diagrams.

${ }^{31}$ G. Baym, in Mathematical Methods in Solid State and Superfluid Theory, Scottish Universities' Summer School 1967, R.C. Clark and G.H. Derrick, Eds. (Oliver \& Boyd, Edinburgh, 1967), p. 121.

32 J. Annett, N. Goldenfeld, and S.R. Renn, Phys. Rev. B 43, 2778 (1991).

${ }^{33}$ C. Panagopoulos and T. Xiang, Phys. Rev. Lett. 81, 2336 (1998).

${ }^{34}$ When the particle density $n$ is evaluated by including fluctuation corrections (over and above the BCS contribution) to the diagonal single-particle Green's function, (one-half of) the (fermionic) particle density is no longer identified with the condensate density in the strong-coupling limit, but rather with the sum of the (bosonic) condensate and noncondensate densities [P. Pieri, L. Pisani, and G.C. Strinati (unpublished)].

${ }^{35}$ S. T. Beliaev, Sov. Phys.-JETP 7, 299 (1958).

${ }^{36}$ V.N. Popov, Functional Integrals and Collective Excitations (Cambridge University Press, Cambridge, 1987).

${ }^{37}$ M. Marini, F. Pistolesi, and G.C. Strinati, Eur. Phys. J. B 1, 151 (1998).

38 T. Kostyrko and R. Micnas, Phys. Rev. B 46, 11025 (1992).

${ }^{39}$ C. Panagopoulos et al., Phys. Rev. B 64, 094514 (2001).

${ }^{40}$ L. Benfatto, S. Caprara, C. Castellani, A. Paramekanti, and M. Randeria, Phys. Rev. B 63, 174513 (2001); L. Benfatto, A. Toschi, S. Caprara, and C. Castellani Phys. Rev. B 64, 140506 (2001).

${ }^{41}$ Q. Chen, I. Kosztin, B. Jankó, and K. Levin, Phys. Rev. Lett. 81, 4708 (1998).

42 The bosonic current correlation function within the Bogoliubov approximation can alternatively be obtained by exploiting its Lehmann representation, for which the relevant matrix elements of the current operator can be explicitly constructed (cf. Ref. 22).

${ }^{43}$ F. De Pasquale and E. Tabet, Ann. Phys. 51, 223 (1969).

${ }^{44}$ It has been assumed that the bosonic interaction potential $V_{B}(q)$ is approximately constant in the range $0 \leq q \lesssim$ $\xi_{\text {phase }}^{-1}$, so that $V_{B}(q)$ remains positive in that range.

${ }^{45}$ M.R. Schafroth, Phys. Rev. B 100, 463 (1955).

${ }^{46} K(\infty)$ becomes negative when $n_{B} \xi_{\text {phase }}^{3}<\left(36 \pi^{2}\right)^{-1} \simeq$ $2.8 \times 10^{-3}$, thus violating the criterion for the occurrence of the Meissner effect. From the relation $n_{B} \xi_{\text {phase }}^{3}=$ $\left(16 \pi n_{B}^{1 / 3} a_{B}\right)^{-3 / 2}$ (obtained from Eq. (C8) with $n_{0}=n_{B}$ and $V_{B}(0)=4 \pi a_{B} / m_{B}, a_{B}$ being the bosonic scattering length), the above upper value for $n_{B} \xi_{\text {phase }}^{3}$ is seen to coincide with the value at which the gas parameter $n_{B}^{1 / 3} a_{B}$ becomes unity, thus violating the diluteness condition. 